\title{
Endothelial miR-30c suppresses tumor growth via inhibition of TGF- $\beta$-induced Serpine1
}

\author{
James V. McCann, ${ }^{1}$ Lin Xiao, ${ }^{2}$ Dae Joong Kim, ${ }^{3}$ Omar F. Khan, ${ }^{4,5}$ Piotr S. Kowalski, ${ }^{4}$ Daniel G. Anderson, ${ }^{4,5,6,7}$ Chad V. Pecot, ${ }^{8,9}$ \\ Salma H. Azam, ${ }^{10}$ Joel S. Parker, ${ }^{8,9,10}$ Yihsuan S. Tsai, ${ }^{8}$ Alisa S. Wolberg, ${ }^{11}$ Stephen D. Turner, ${ }^{12,13}$ Kohei Tatsumi, ${ }^{14}$ \\ Nigel Mackman, ${ }^{14}$ and Andrew C. Dudley ${ }^{3,15}$ \\ 'Department of Cell Biology and Physiology, University of North Carolina (UNC) at Chapel Hill, Chapel Hill, North Carolina, USA. Children's Cancer Institute, Kensington, New South Wales, Australia. \\ ${ }^{3}$ Department of Microbiology, Immunology, and Cancer Biology, The University of Virginia, Charlottesville, Virginia, USA. ${ }^{4}$ David H. Koch Institute for Integrative Cancer Research, Massachusetts Institute of \\ Technology (MIT), ${ }^{5}$ Department of Chemical Engineering, ${ }^{6}$ Harvard-MIT Division of Health Sciences and Technology, and 'Institute for Medical Engineering and Science, MIT, Cambridge, Massachusetts, USA. \\ ${ }^{8}$ Lineberger Comprehensive Cancer Center, ${ }^{9}$ School of Medicine, ${ }^{10}$ Department of Cenetics, and "Department of Pathology and Laboratory Medicine, UNC McAllister Heart Institute, UNC at Chapel Hill, Chapel \\ Hill, North Carolina, USA. ${ }^{2}$ Department of Public Health Sciences, and ${ }^{13}$ Bioinformatics Core, University of Virginia School of Medicine, Charlottesville, Virginia, USA. ${ }^{14}$ Department of Medicine, Division of \\ Hematology and Oncology, UNC McAllister Heart Institute, UNC at Chapel Hill, Chapel Hill, North Carolina, USA. ${ }^{55}$ Emily Couric Cancer Center, The University of Virginia, Charlottesville, Virginia, USA.
}

In tumors, extravascular fibrin forms provisional scaffolds for endothelial cell (EC) growth and motility during angiogenesis. We report that fibrin-mediated angiogenesis was inhibited and tumor growth delayed following postnatal deletion of Tgfbr2 in the endothelium of Cdh5-Cre ${ }^{E R T 2} \mathrm{Tgfbr}^{f / f f l}$ mice (Tgfbr ${ }^{i E C K O}$ mice). ECs from Tgfbr2 ${ }^{i E C K O}$ mice failed to upregulate the fibrinolysis inhibitor plasminogen activator inhibitor 1 (Serpine1, also known as PAI-1), due in part to uncoupled TCF- $\beta$-mediated suppression of miR-30c. Bypassing TCF- $\beta$ signaling with vascular tropic nanoparticles that deliver miR-30c antagomiRs promoted PAI-1-dependent tumor growth and increased fibrin abundance, whereas miR30c mimics inhibited tumor growth and promoted vascular-directed fibrinolysis in vivo. Using single-cell RNA-Seq and a NanoString miRNA array, we also found that subtypes of ECs in tumors showed spectrums of Serpine1 and miR-30c expression levels, suggesting functional diversity in ECs at the level of individual cells; indeed, fresh EC isolates from lung and mammary tumor models had differential abilities to degrade fibrin and launch new vessel sprouts, a finding that was linked to their inverse expression patterns of miR-30c and Serpine1 (i.e., miR-30c ${ }^{\text {hi }}$ Serpine ${ }^{10}$ ECs were poorly angiogenic and $\mathrm{miR}-30 \mathrm{c}^{\mathrm{lo}}$ Serpine $\mathrm{1}^{\mathrm{hi}}$ ECs were highly angiogenic). Thus, by balancing Serpine1 expression in ECs downstream of TCF- $\beta$, $\mathrm{miR}-30 \mathrm{c}$ functions as a tumor suppressor in the tumor microenvironment through its ability to promote fibrin degradation and inhibit blood vessel formation.

\section{Introduction}

To support their growth, cancer cells recapitulate latent developmental or physiological processes including those important for tissue repair, wound healing, and angiogenesis; however, reactivation of these processes is typically carried out in an exaggerated and uncoordinated manner. A good example is the co-option and subversion of the different phases of wound healing, which has long been recognized as a cardinal feature of solid tumors (1). In both healing wounds and tumors, fragile and leaky endothelium instigates the formation of a fibrin plug that seals the vasculature and prevents blood loss. Granulation tissue consisting of platelets along with inflammatory cells reinforces the fibrin plug and helps to orchestrate the mobilization and activation of (myo)fibroblasts that aid in tissue repair and development of new blood vessels that

Conflict of interest: DCA has applied for a patent on 7C1 entitled "Conjugated lipomers and uses thereof" (US patent application no. 9238716). Copyright: (c) 2019 American Society for Clinical Investigation Submitted: June 25, 2018; Accepted: February 1, 2019 Reference information: / Clin Invest. 2019;129(4):1654-1670. https://doi.org/10.1172/JCI123106. provide oxygen and nutrients and remove waste products (2). The patterns of gene expression in tumors and healing wounds are remarkably similar to the extent that a "wound signature" is recognized to have prognostic value in predicting poor patient survival and cancer progression $(3,4)$. Despite the similarities, there are notable differences; for example, endothelial cells (ECs) express several unique factors that can distinguish physiological (wound) from pathological (tumor) angiogenesis (5).

While wound healing is an acute, self-limiting, and tightly regulated process, the "tumor wound" is chronic and proceeds without normal feedback mechanisms. For example, new tumor blood vessels are dysfunctional and persistently hyperpermeable, leading to perivascular fibrin(ogen) deposition $(1,6,7)$. Fibrinogen that escapes leaky tumor vasculature is rapidly converted to an insoluble fibrin meshwork that is cross-linked and stabilized by factor XIII. Given its abundant and near-ubiquitous presence in solid tumors, probes that can detect fibrin or fibrin-fibronectin complexes have recently been used in imaging modalities for various cancers and their metastases (8). Fibrin forms a provisional scaffold for angiogenesis, it sequesters growth factors such as VEGF and TGF- $\beta$, and it is degraded by plasmin, a serine protease that is 
generated from the zymogen plasminogen by key components of the plasminogen-activating system including tissue plasminogen activator (tPA), urokinase plasminogen activator (uPA), and urokinase plasminogen activator receptor (uPAR). Fibrin degradation liberates growth factors and generates fibrin degradation products that themselves can serve as a serum biomarker to detect tumors of various origins.

Inhibition of fibrin degradation is mediated in part by plasminogen activator inhibitor 1 (Serpine1, also known as PAI-1), which binds to and inactivates uPA and tPA causing the internalization of UPA via UPAR and LDL receptor-related protein 1 (LRP1) (9). Notably, PAI-1 is enriched in and actively secreted by the endothelium, prompting its designation as "endothelial PAI-1" or "Serpin E1." PAI-1 is overexpressed in multiple cancers, especially breast cancer, in which its higher expression predicts worse overall survival, development of metastases, and poor responses to chemotherapy (10). Despite established associations between overexpression of PAI-1 and tumor progression, its role in solid tumors remains controversial and context dependent. For example, blocking PAI-1 using pharmacological inhibitors diminishes the growth of most tumors, but other tumor types are not affected $(9,11-15)$. Likewise, several studies in PAI-1-KO mice demonstrated growth inhibition of engrafted tumors, whereas other studies showed no obvious effect $(9,16-20)$. These opposing outcomes may be related to the dose-dependent effects of PAI- 1 in different types of cells in the tumor microenvironment (TME), local versus systemic inhibition of PAI-1 itself, and expression and bioavailability of TGF- $\beta$, the major transcriptional activator of Serpine1.

The regulation of Serpine1 by TGF- $\beta$ and its major downstream effectors (SMADs) is well characterized (21). Upon TGF- $\beta$ binding of the type 2 receptor, the recruited type 1 receptor phosphorylates and thereby activates receptor-associated SMADs (RSMADs) such as SMAD2 and SMAD3. These RSMADs form complexes with SMAD4 and, in cooperation with additional cofactors, rapidly upregulate Serpine1 mRNA $(22,23)$. Apart from transcriptional regulation of Serpine1 mRNA production by TGF- $\beta$, it was recently shown that several miRNAs, in particular the miR-30 family, also regulate Serpine1 expression by binding directly to its 3'-UTR. In a study using human vascular ECs, the upregulation of Serpine1 by proangiogenic placental growth factor (PLGF) was augmented when miR-30c was blocked (24). Moreover, PLGF and TGF- $\beta$ were shown to downregulate miR-30c and miR-30d expression in ECs and non-ECs, an event that was linked to several pathological conditions including fibrosis and thrombosis (24-26). While miR-30c itself was recently associated with cancer progression (e.g., breast cancer), in which it serves as a prognostic biomarker in tumor tissue and plasma, no studies have identified a specific role for miR-30c in the TME; in particular, the function of miR-30c and its regulation by TGF- $\beta$ in the tumor vasculature are entirely unknown (27-29).

Here, we used mice with targeted disruption of Tgfbr 2 in the endothelium to explore tumor growth and blood vessel maturation in the absence of TGF- $\beta$ signaling. By screening the functional properties of isolated EC subtypes and their TGF- $\beta$ responses ex vivo, we have identified a vascular-directed pathway, driven by an axis between TGF- $\beta$, miR-30c, and Serpine1, that links a tumor-induced fibrinolytic pathway in the endothelium with tumor progression. Furthermore, we have uncovered an unantic- ipated functional diversity in subtypes of ECs in tumors that can be attributed to expression patterns of $\mathrm{miR}-30 \mathrm{c}$ and Serpine1 that is also associated with clinical outcomes in breast cancer patients. Our findings shed light on the specific functional role of TGF- $\beta$ in the tumor vasculature and identify what we believe to be new tumor-supportive properties for the highly specialized, aberrant, and heterogeneous tumor endothelium (30-32).

\section{Results}

Postnatal deletion of Tgfbr 2 in endothelium delays tumor growth and impairs blood vessel formation. TGF- $\beta$ plays a well-defined role in the promotion of fibrosis, tissue scarring, and wound healing as a result of its ability to activate multiple genes important for cell motility, survival, and coagulation and hemostasis (33). However, the function of TGF- $\beta$ in pathophysiological processes such as tumor growth is challenging to study in a cell-type-specific manner in vivo and requires, for example, conditional deletion strategies using lineage-specific Cre drivers (34). Furthermore, because TGF- $\beta$ is reported to have both pro- and antiangiogenic activities in ECs that are dose and context dependent, the precise function of TGF- $\beta$ in the vasculature during tumor growth and progression is unclear (35-37). To define the role of TGF- $\beta$ in the endothelium during tumor growth, we generated mice with conditional deletion of Tgfbr2 by crossing Cdh5-Cre $E^{E R T 2}$ mice with $T g f b r 2^{f l / l}$ mice

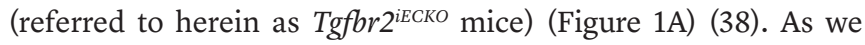
described previously, in some of these mice we also bred in the Ai6 ZSGreen reporter to facilitate EC isolation, to confirm EC-specific Cre activity, and to demonstrate disruption in TGF- $\beta$ signaling in ECs from engrafted tumors (Supplemental Figure 1, A and B; supplemental material available online with this article; https://doi. org/10.1172/JCI123106DS1) (39). Because Tgfbr2 is required for signaling following its dimerization with TGF- $\beta$ R1, the resultant Tgfbr $2^{i E C K O}$ mice show disabled TGF- $\beta$ signaling in the endothelium in response to all 3 TGF- $\beta$ ligands following tamoxifen administration. After generating these mice and control mice (also treated with tamoxifen), we orthotopically engrafted syngeneic EO771 mammary tumor cells into the mammary fat pad and then measured tumor volumes daily. The results showed that tumor sizes were smaller and tumor volumes were reduced by 1.8 -fold (746.7 $\pm 46.7 \mathrm{~cm}^{3}$ versus $\left.400.2 \pm 74.1 \mathrm{~cm}^{3}\right)$ and that tumor weights were reduced by 1.6 -fold $(1.1 \pm 0.14 \mathrm{~g}$ versus $0.65 \pm 0.1 \mathrm{~g})$ in $\mathrm{Tg} f b r 2^{\text {iЕСКО }}$ mice compared with control mice (Figure 1B). CD31 staining for blood vessel networks revealed that tumors in $\mathrm{Tg} f b r 2^{i \text { IEКO }}$ mice had a 2.4-fold reduction in total vascular area along with a 3.1-fold reduction in open vessel lumens compared with controls (Figure 1, C and D). Furthermore, using an automated software platform to analyze vessel branching and complexity, we observed a reduction in the total number of vessel branches when normalized to tumor area and a reduction in branch length when distributed by frequency in Tgfbr ${ }^{\text {iECKO }}$ tumors versus controls (Figure 1, E and F). In H\&E-stained paraffin sections, we also observed less amorphous pink-colored material, verified to be fibrin(ogen) that had escaped leaky vasculature, in tumors from $\mathrm{Tg} f b r 2^{i E C K O}$ mice compared with those from control mice (data not shown and Supplemental Figure 2, A and B). Taken together, these results suggest that TGF- $\beta$ signaling is important for the development of tumor blood vessels that support primary mammary tumor growth. 
A
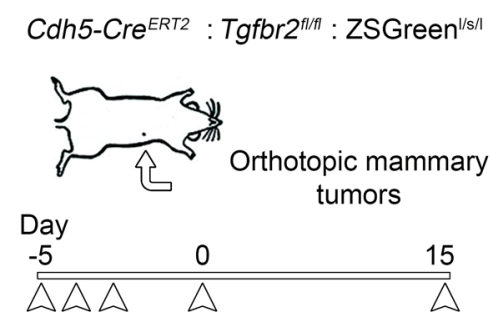

Tamoxifen Inject Harvest
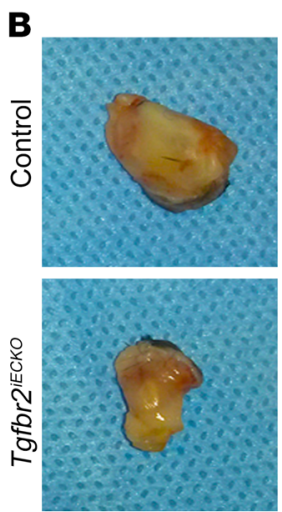

Tgfbr2iECKO

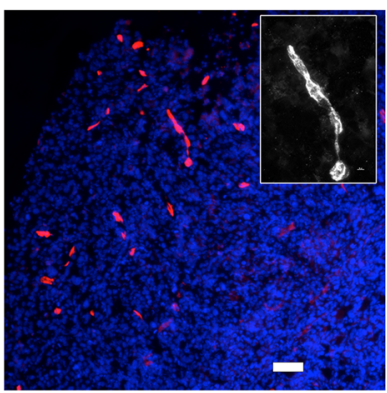

Control

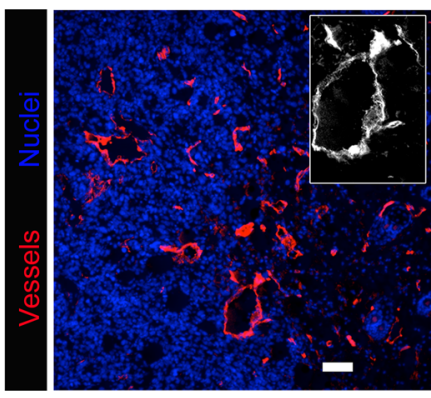

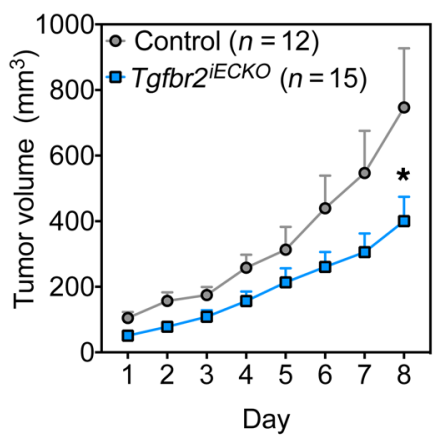

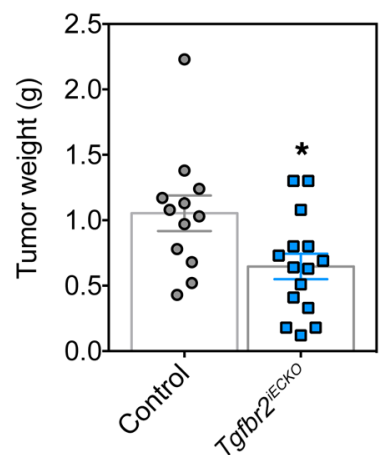

D
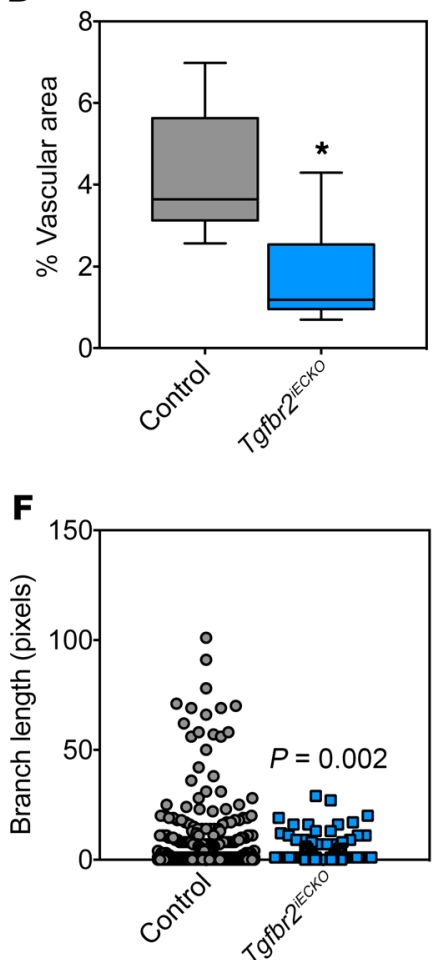
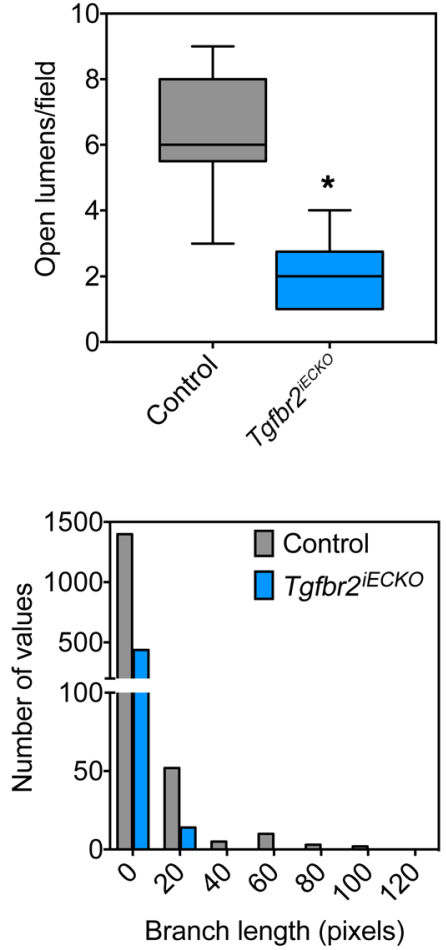

$\mathbf{E}$

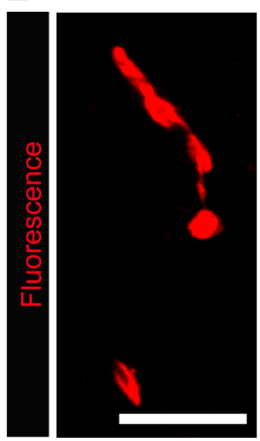

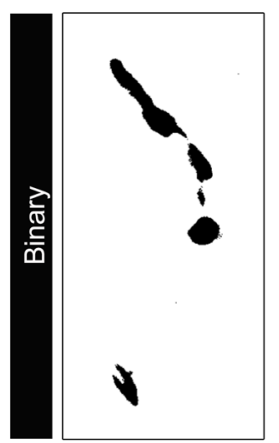

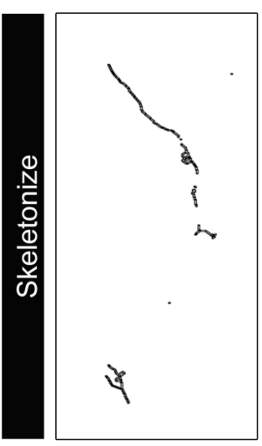

Figure 1. Postnatal deletion of Tgfbr2 in endothelium delays tumor growth and impairs blood vessel formation. (A) Study design including tamoxifen treatment and orthotopic mammary tumor injection schedule for control versus $\mathrm{Tg} f b r 2^{\mathrm{iECKO}}$ mice. (B) Mammary tumors from control mice versus those from $T g f b r 2^{i E C K O}$ mice. Tumor volumes were determined with calipers using the formula: volume $=\left(\right.$ length $\times$ width $\left.{ }^{2}\right) / 2$. Final tumor weights are presented in grams (control, $n=12$; Tgfbri ${ }^{i E C K O}, n=15$ ). ${ }^{*} P<0.05$, by ANOVA with Sidak's multiple comparisons test (volume) and Student's $t$ test (weight). (C) CD31 staining of control versus Tgfbri ${ }^{i E C K O}$ mammary tumors. The inset images show a representative open or closed lumen of a blood vessel in each tumor. Scale bars: 100 $\mu \mathrm{m} ;$ zoom, $\times 4$ (insets). (D) Percentage of vascular area and number of open lumens per field in tumors from control versus Tgfbr $2^{\mathrm{iECKO}}$ mice $(n=5$ tumors examined per group). ${ }^{*} P<0.05$, by Student's $t$ test. (E) Image analysis scheme to determine branch lengths in tumors from control versus Tgfbr2iEcKo mice. See Methods for analysis details. The representative vessel is from the far-right inset in C. Scale bar: $100 \mu \mathrm{m}$. (F) Quantification of branch lengths in control versus Tgfbr2iECKO tumors using the "analyze skeleton" feature in Imagej Fiji (see Methods for details). Results were determined by Mann-Whitney $U$ test. Histogram analysis of branch length shows the number of branches binned per 20-pixel interval ( $n=3$ tumors per group). Data represent the mean \pm SEM.

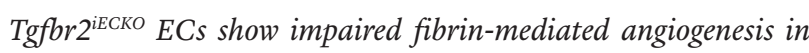
vivo and in vitro and fail to upregulate Serpine1 and downregulate miR-30 miRNAs. Next, we isolated primary ECs from the lungs of $T g f b r 2^{i Е C К O}$ and WT mice to examine their in vitro responses to TGF- $\beta$. After preparing several EC isolates from $\mathrm{Tg} f b r 2^{i E C K O}$ mice, we performed FACS and quantitative PCR (qPCR) to identify an isolate with complete disruption of Tgfbr2 (40). As we have shown previously, expression of the ZSGreen reporter, enrichment for bona fide EC marker mRNAs, and positive uptake of Dil-Ac-LDL along with CD31 (also known as PECAM) expression confirmed EC purity (Figure 2, A and B, and Supplemental Figure 3A) (39).

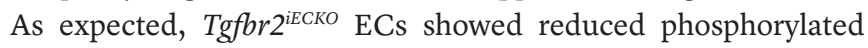
SMAD2 (p-SMAD2) upon TGF- $\beta$ stimulation, confirming that TGF- $\beta$ signaling was disabled (Figure $2 \mathrm{C}$ ). To assess the in vivo and in vitro angiogenic potential of $\mathrm{Tg} f b r 2^{i E C K O} \mathrm{ECs}$ versus control ECs, we performed fibrin plug assays in syngeneic mice and sprout- 
A
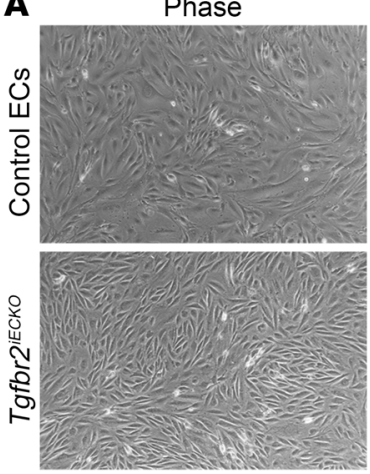

ZsGreen

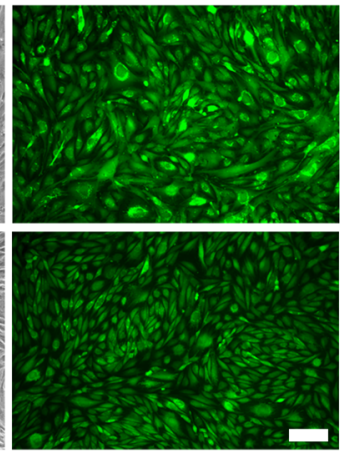

B

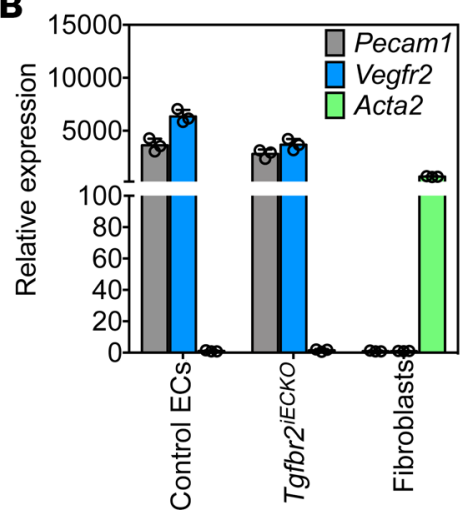

C

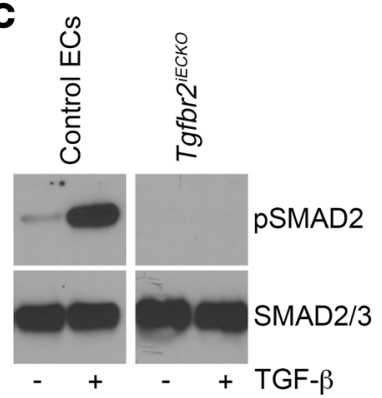

D

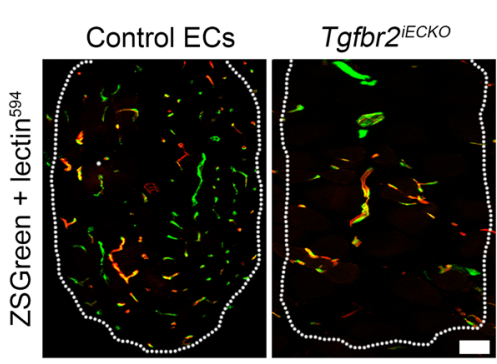

Fibrin plug (in vivo)

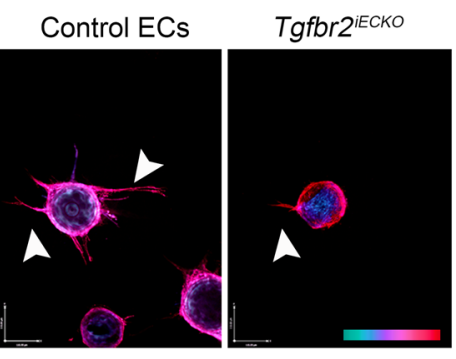

Fibrin gel (in vitro)
E

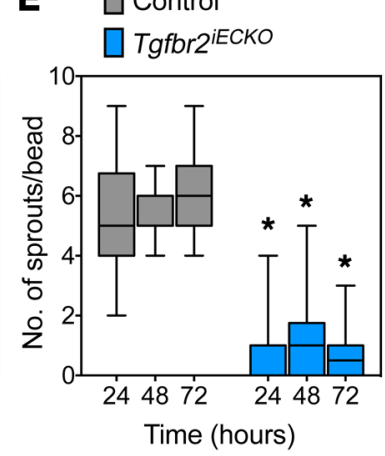

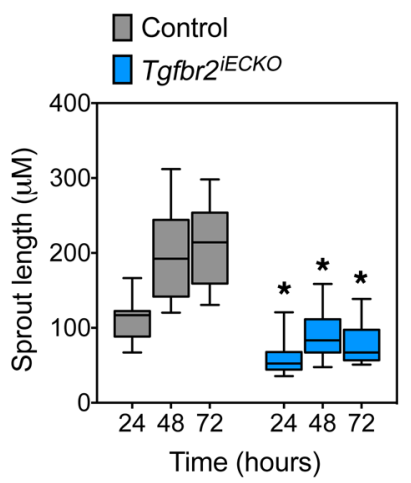

$\mathbf{F}$

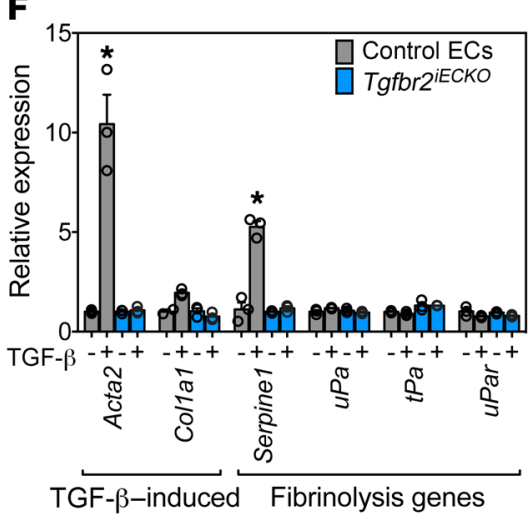

G

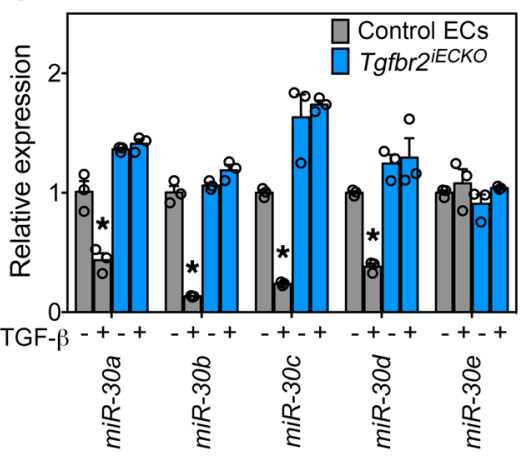

H

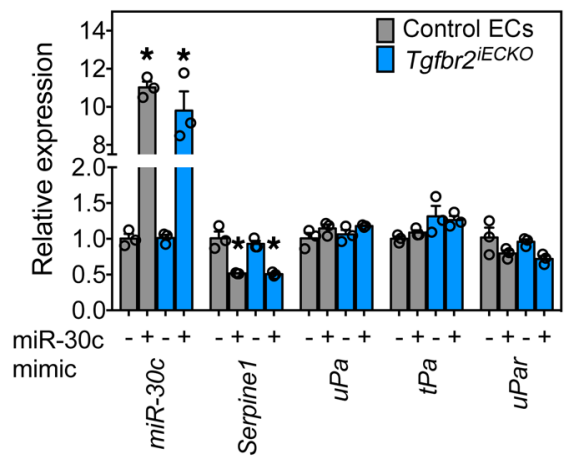

Figure 2. Tgfbr ${ }^{i \in c k o}$ ECs show impaired fibrin-mediated angiogenesis in vitro and fail to upregulate Serpine1 and downregulate miR-30 miRNAs. (A) Validation of EC isolation from ZSGreen reporter mice. Phase and ZSGreen images of isolated ECs. Scale bar: $10 \mu \mathrm{m}$. (B) qPCR analysis of EC and fibroblast genes from control and Tgfbr2iECKo mice. Samples were assayed in triplicate. (C) Western blot showing phosphorylated SMAD2 (p-SMAD2) in ECs challenged with 10 $\mathrm{ng} / \mathrm{ml}$ TGF- $\beta$ to confirm disabled Tgfbr2 signaling. (D) In vivo fibrin plugs from control versus Tgfbr ${ }^{\text {iECKo }}$ ECs. Lectin ${ }^{594}$ was injected prior to euthanasia. Scale bar: $100 \mu \mathrm{m}$. Phalloidin-stained ECs in the fibrin bead-sprouting assay at 72 hours. Arrows indicate examples of individual sprouts that were quantified. The scale at bottom right indicates sprout depth in the 3D fibrin matrix. Scale bars: $40 \mu \mathrm{m}(x)$ and $54 \mu \mathrm{m}(y)$. (E) Quantification of the fibrin-sprouting assay over time. The number and length of sprouts were measured for each indicated time point ( $n=30$ beads per time point). (F) qPCR analysis of TCF- $\beta$-induced and fibrinolysis-regulating genes in control versus $\mathrm{Tgfbr}^{\mathrm{iECKO}} \mathrm{ECs}$. Samples were assayed in triplicate. (C) miR-30 family miRNA expression in ECs treated with 10 $\mathrm{ng} / \mathrm{ml} \mathrm{TGF-} \beta$ for 48 hours. Samples were assayed in triplicate. (H) Ectopic miR-30c mimic transfection and qPCR analysis of miR-30c levels along with fibrinolysis-regulating genes. Samples were assayed in triplicate. ${ }^{*} P<0.05$, by ANOVA $(\mathbf{E})$ and Student's $t$ test $(\mathbf{F}-\mathbf{H})$ Data represent the mean \pm SEM.

ing assays in fibrin gels (41). By perfusing mice with lectin ${ }^{594}$ just before euthanasia, we could distinguish host-derived (ZSGreen lectin ${ }^{594+}$ ) ECs from engrafted (ZSGreen ${ }^{+}$lectin ${ }^{594+}$ ) ECs. Consistent with what we observed in Tgfbr ${ }^{i E C К O ~ m i c e, ~ t h e ~ e n g r a f t e d ~}$ control ECs formed greater numbers of vessels compared with their KO counterparts; furthermore, we found that control ECs launched numerous sprouts per bead $(6.3 \pm 0.3$ sprouts/ bead $)$ in vitro, whereas $\mathrm{Tg} f b r 2^{i E C K O}$ ECs were deficient in their ability to form new sprouts in fibrin (0.7 \pm 0.2 sprouts/bead) (Figure 2, D and E). Sprout lengths were 2.7-fold longer at 72 hours in control ECs compared with those in ECs from their Tgfbr $2^{i E C K O}$ counterparts $(211.5 \pm 11.9 \mu \mathrm{m}$ versus $78.5 \pm 5.9 \mu \mathrm{m})$, suggesting that intact TGF- $\beta$ signaling in ECs is required to promote the formation of mature vascular structures in a fibrin matrix. 
A

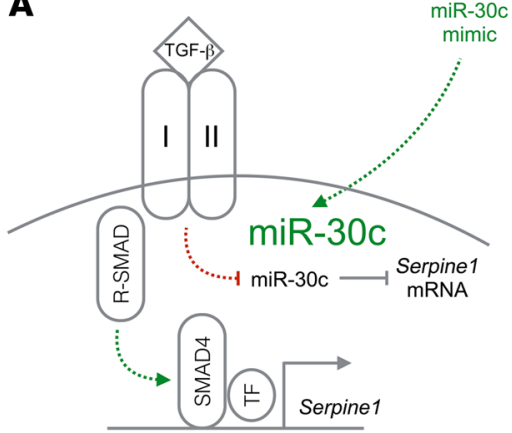

B

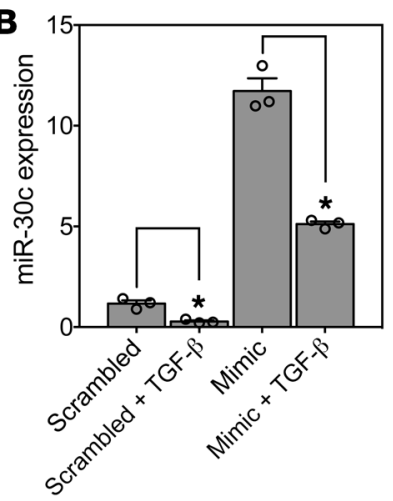

C

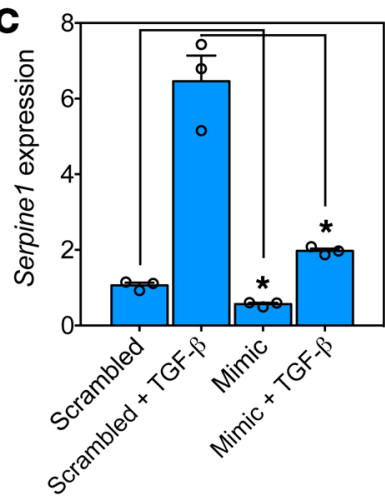

D

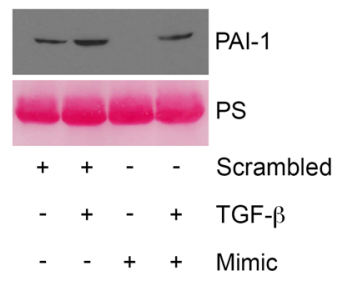

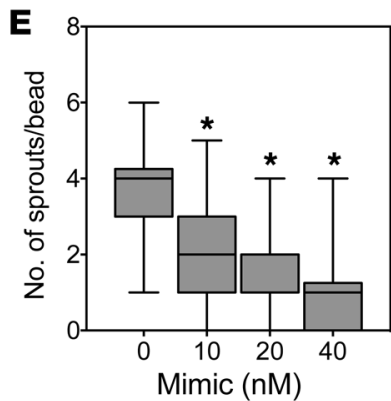

G

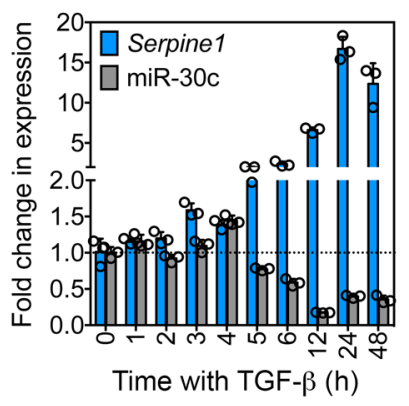

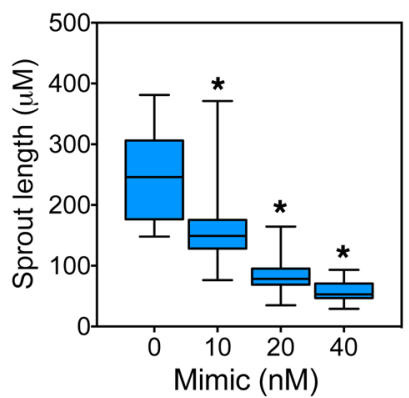

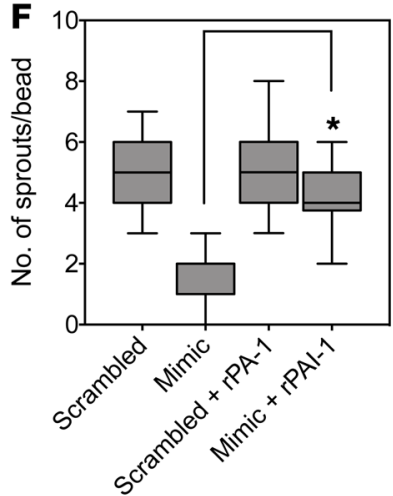

I

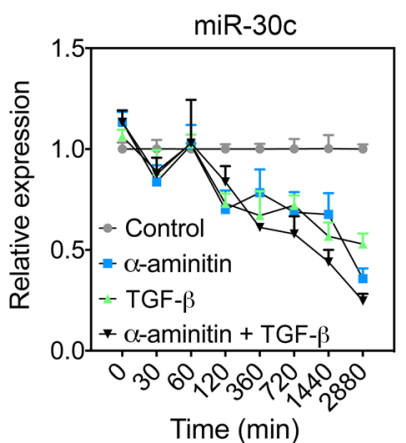

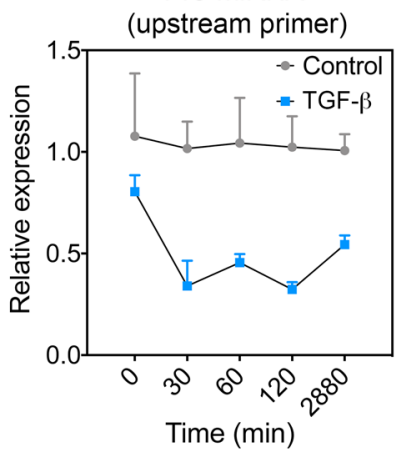

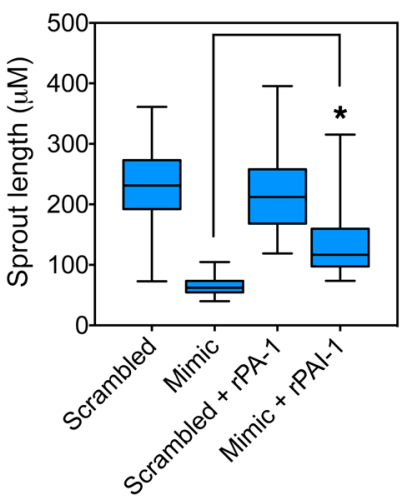

Pre-miRNA (downstream primer)

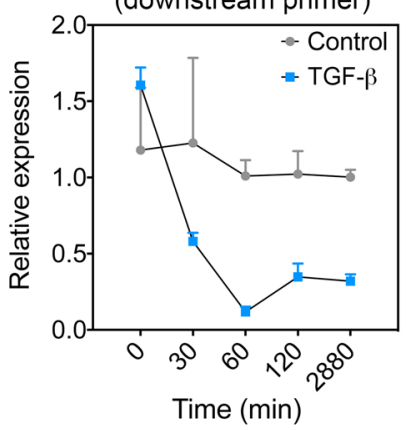

Figure 3. miR-30c mimics block Serpine1 expression and inhibit in vitro EC sprouting. (A) Two pathways to Serpine1 induction by TCF- $\beta$ signaling in ECs. (B) qPCR analysis of miR-30c in ECs treated with $10 \mathrm{ng} / \mathrm{ml} \mathrm{TCF-} \beta, 20 \mathrm{nM}$ miR-30c mimic, or a combination of both. Samples were assayed in triplicate $(n=3)$. (C) qPCR analysis of Serpine1 in ECs treated with $10 \mathrm{ng} / \mathrm{ml} \mathrm{TGF}-\beta, 20 \mathrm{nM}$ miR-30c mimic, or the combination. Samples were assayed in triplicate $(n=3)$. (D) Western blot analysis of PAl-1 in ECs treated with $10 \mathrm{ng} / \mathrm{ml} \mathrm{TCF}-\beta, 20 \mathrm{nM}$ miR-30c mimic, or a combination of both. Ponceau staining (PS) was used to show equal loading. (E) Fibrin bead-sprouting assay in ECs treated with miR-30c mimic for 72 hours $(n=30$ beads). Controls were treated with a 40-nM scrambled sequence. (F) Number and length of sprouts in ECs treated with miR-30c mimic, rPAl-1 ( $0.8 \mu \mathrm{g} / \mathrm{ml})$, or a combination of both for 72 hours ( $n=30$ beads). (G) qPCR analysis of ECs treated with $10 \mathrm{ng} / \mathrm{ml} \mathrm{TCF}-\beta$ for each indicated time point. Samples were assayed in triplicate $(n=3)$. (H) qPCR analysis of ECs treated with $10 \mathrm{ng} / \mathrm{ml} \mathrm{TGF-} \beta, 10 \mu \mathrm{M} \alpha$-amanitin, or a combination of both. Samples were assayed in triplicate $(n=3)$. (I) qPCR analysis of pre-miRNA for miR-30c in ECs treated with $10 \mathrm{ng} / \mathrm{ml}$ TGF- $\beta$ for the indicated durations. Samples were assayed in triplicate $(n=3)$. ${ }^{*} P<0.05$, by Student's $t$ test $(\mathbf{B}$ and $\mathbf{C})$, ANOVA $(\mathbf{E})$, and Student's $t$ test $(\mathbf{F})$. Data represent the mean $\pm \mathrm{SEM}$.

Because these in vitro sprouting assays are performed in a fibrin-rich microenvironment similar to that found in solid tumors, we hypothesized that $T g f b r 2^{\text {iECKO }}$ ECs had enhanced fibrinolytic activity, resulting in loss of the fibrin scaffolds that are required to produce stable vessels $(42,43)$. By using qPCR to measure the expression of TGF- $\beta$-induced genes known to control fibrinolysis, we found that the fibrinolysis inhibitor Serpine1 was the only gene

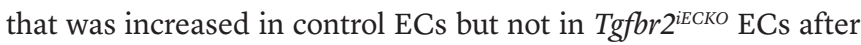
TGF- $\beta$ challenge (Figure 2F). Acta 2 and Col1a 1 were used as TGF- $\beta$ regulated positive controls. Furthermore, fibrin zymography gels revealed that control ECs had reduced fibrin degradation when challenged with TGF- $\beta$, whereas Tgfbr2 $2^{\text {iECKO }}$ ECs did not (Supplemental Figure 3B). Thus, impaired tumor growth and angiogenesis

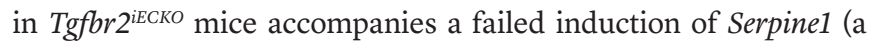
major vascular-enriched inhibitor of plasmin generation and thus fibrin degradation) in ECs that is necessary for balancing the rate of fibrinolysis and fibrin-mediated vessel maturation in the TME.

Recently, it was suggested that members of the miR-30 family of miRNAs target the Serpine1 gene and regulate EC morphogenesis $(44,45)$; however, links between TGF- $\beta$, miR-30c, Serpine1, 
and fibrin-mediated angiogenesis have not been elucidated. Thus, we hypothesized that in addition to the well-characterized SMAD-dependent regulation of Serpine1, TGF- $\beta$ may also regulate endothelial Serpine1 expression via a miR-30-dependent mechanism that controls sprouting angiogenesis. First, we used qPCR to measure the levels of miR-30 family members in control

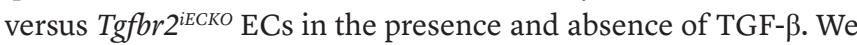
observed that TGF- $\beta$ strongly reduced the expression of miR-30 miRNAs (with the exception of miR-30e) in control ECs but not in Tgfbr ${ }^{i E C K O}$ ECs (Figure 2G). Because miR-30c is reported to have the highest degree of species conservation with respect to binding sites in the 3'-UTR of Serpine1, we turned our attention to miR$30 \mathrm{c}$ for subsequent analysis (24). Ectopic expression of miR-30c

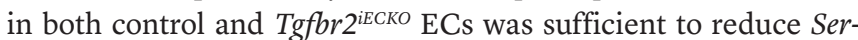
pine 1 mRNA expression by $50 \%$, but other well-known fibrinolysis genes including $u \mathrm{~Pa}, t \mathrm{~Pa}$, and $u$ Par were not affected (Figure $2 \mathrm{H}$ ).

miR-30c mimics block Serpine1 expression and inhibit in vitro EC sprouting. Next, we tested whether miR-30c was capable of regulating TGF- $\beta$-induced Serpine1 expression in ECs in parallel to the wellknown SMAD-dependent regulation of Serpine1 (Figure 3A). Ectopic expression of miR-30c was reduced by TGF- $\beta$ addition and was sufficient to abrogate TGF- $\beta$-induced Serpine1 (and PAI- 1 secretion) in ECs (relative to TGF- $\beta$-treated controls) (Figure 3, B-D). TGF- $\beta$ mediated reduction in miR-30c was dose dependent, was not similarly regulated by other angiogenic factors including VEGF and basic fibroblast growth factor (bFGF), and was independent of SMAD4 (a co-SMAD that is typically needed for RSMADs to properly function) and Eri-1 (an exoribonuclease suggested to negatively regulate the abundance of some mature miRNAs) (Supplemental Figure 4, A-C) $(33,46)$. Ectopic miR-30c expression was also sufficient to dosedependently reduce sprout numbers and length in fibrin-mediated angiogenesis assays in vitro, but this effect was rescued by adding exogenous recombinant PAI-1 (rPAI-1) (Figure 3, E and F, and Supplemental Figure 5A). As expected, silencing Serpine1 using siRNAs phenocopied the effect of the miR-30c mimic, as it reduced sprout numbers and length (Supplemental Figure 5, B and C); taken together, miR-30c controls Serpine1 expression downstream of TGF- $\beta$, and an axis between these 3 factors may function as an important "rheostat" for controlling the rate of vascular-directed fibrin generation and degradation and vessel morphogenesis.

To begin to understand the mechanism whereby TGF- $\beta$ might regulate $\mathrm{miR}-30 \mathrm{c}$, we examined the temporal expression patterns of miR-30c and Serpine1. We found that the reduction in miR-30c by TGF- $\beta$ generally coincided inversely with the increase in Serpine1, suggesting that miR-30c processing or transcription might be affected (Figure 3G). Indeed, miR-30c expression was reduced with similar kinetics by the Pol2 inhibitor $\alpha$-amanitin in both the presence and absence of TGF- $\beta$ (Figure $3 \mathrm{H}$ ). Using primers specific for the miR-30c pre-miRNA coding sequence found within chromosome 1 , we found that TGF- $\beta$ resulted in a time-dependent reduction in the generation of the pre-miRNA for miR-30c, which preceded the reduction in miR-30c transcript expression (Figure 3I). Of note, these results do not conflict with the recently described targetdirected miRNA degradation mechanism whereby Serpine1 mRNA can itself control the expression of miR-30c after serum stimulation (47). Thus, TGF- $\beta$ appears to interfere with the transcriptional pathway that generates mature miR-30c from pre-miRNA precursors.
miR-30c gain of function in the endothelium inhibits tumor growth and reduces fibrin abundance, whereas $m i R-30 c$ loss of function does the opposite. To assess the function of miR-30c in tumor-associated ECs (TECs) in vivo, we used vascular tropic 7C1 nanoparticles that efficiently deliver nucleic acid payloads to the vasculature in tumor models $(48,49)$. We chose the E0771 mammary tumor cell line that is low or absent for Serpine1 mRNA expression and PAI-1 secretion, even when challenged with TGF- $\beta$ in vitro (Figure 4 , A and B). Thus, ECs are probably the predominant source of locally acting PAI-1 in these tumors in vivo. After injecting EO771 tumor cells orthotopically into the mammary fat pad, the mice were treated 3 times per week with 7C1 mimic (miR-30c conjugates) or 7C1 scrambled nanoparticles. The results showed that tumor volumes were reduced by 3.9-fold $\left(308.5 \pm 65.3 \mathrm{~cm}^{3}\right.$ versus $\left.78.5 \pm 16.5 \mathrm{~cm}^{3}\right)$ and final tumor weights by 2.3 -fold $(0.7 \pm 0.2 \mathrm{~g}$ versus $0.3 \pm 0.08 \mathrm{~g})$ in the $7 \mathrm{C} 1 \mathrm{mimic}-$ treated versus $7 \mathrm{C} 1$ scramble-treated mice (Figure $4, \mathrm{C}$ and $\mathrm{D}$ ). Using a similar strategy, we tested how miR-30c loss of function in the endothelium would impact tumor growth. In contrast to ectopic miR-30c expression, 7C1 antagomiRs (anti-miR-30c) strikingly stimulated tumor growth compared with 7C1 scramble-treated control mice. We observed a 4.3-fold increase in overall final tumor volume $\left(1043.2 \pm 162.1 \mathrm{~cm}^{3}\right.$ versus $\left.243.5 \pm 32.5 \mathrm{~cm}^{3}\right)$ and a 2.1 -fold increase in final tumor weight $(1.7 \pm 0.4 \mathrm{~g}$ versus $0.8 \pm 0.3 \mathrm{~g})$ in $7 \mathrm{C} 1$ antagomiR-treated compared with $7 \mathrm{C} 1$ scramble-treated mice (Figure 4, C and D). Notably, we observed no significant change in tumor volumes in the $7 \mathrm{C} 1$ mimic- or $7 \mathrm{C} 1$ antagomiR-treated mice versus the $7 \mathrm{C} 1$ scramble-treated mice on a PAI-1-KO background, suggesting that the impact of miR-30c on tumor growth via the vasculature requires host-derived PAI-1 (Figure 4E).

To ensure that ECs were being targeted in these tumors, we used FACS to isolate and molecularly profile ZSGreen ${ }^{+}$ECs from EO771 tumors injected into the mammary fat pads of $C d h 5$ $\mathrm{Cre}^{E R T 2}$ ZSGreen ${ }^{f l f l}$ mice treated with $7 \mathrm{C} 1$ mimics or 7C1 antagomiRs. ZSGreen- "non-ECs" were collected in tandem as a negative control, and sample purities were confirmed using qPCR (Supplemental Figure 6, A and B). We detected a 63\% reduction in Serpine1 mRNA expression and 5.1-fold increase in miR-30c levels in ECs from the 7C1 mimic-treated versus the 7C1 scramble-treated mice. We observed no difference in expression of miR-30c or Serpine1 in the non-EC fraction (Figure 4, F and G). In contrast, ECs from the 7C1 antagomiR-treated group had a 7.7-fold increase in Serpine1 mRNA and a 2.7-fold decrease in miR-30c expression compared with 7C1 scrambled control ECs. Again, we observed no significant change in expression of Serpine1 or miR-30c in the isolated non-ECs (Figure 4, F and G). The circulating levels of PAI-1 in plasma measured by ELISA were also decreased by 3-fold in the 7C1 mimic-treated mice but were increased by approximately 2 -fold in the $7 \mathrm{C} 1$ antagomiR-treated mice compared with the 7C1 scramble-treated mice, indicating that ectopic manipulation of miR-30c in the vasculature is sufficient to alter PAI-1 secretion by TECs (Figure 4, H and I).

Characterization of blood vessels using immunohistology revealed a 3 -fold reduction in vessel area $(4.5 \% \pm 0.4 \%$ versus $1.4 \% \pm 0.2 \%)$ and a 20 -fold reduction in intratumoral fibrin $(6.2 \%$ $\pm 1.9 \%$ versus $0.3 \% \pm 0.05 \%$ ) in $7 \mathrm{C} 1$ mimic-treated mice (Figure 4 , J and $\mathrm{K}$ ). Thus, ectopic expression of miR-30c in the vasculature reduced intratumoral fibrin abundance, which was accompanied 
A

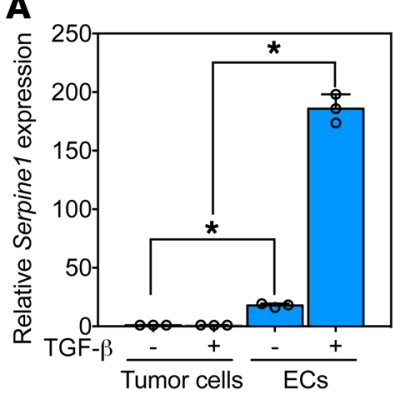

E

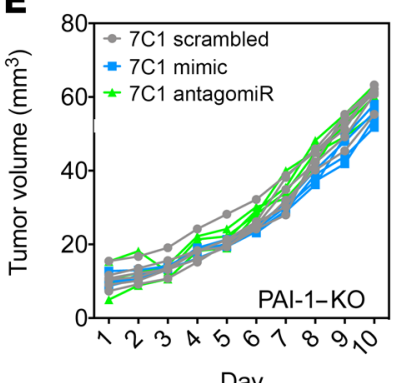

Day

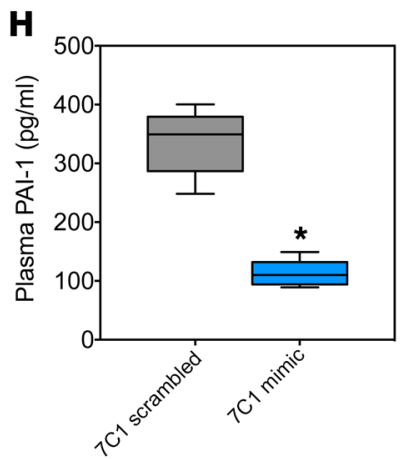

$\mathbf{K}$

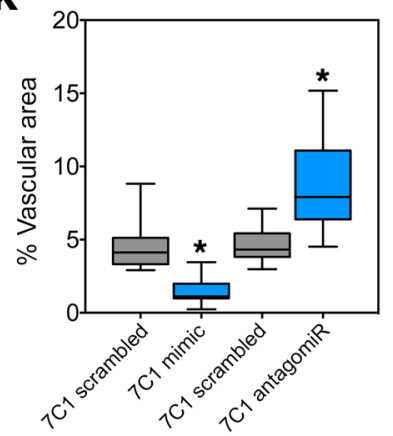

B
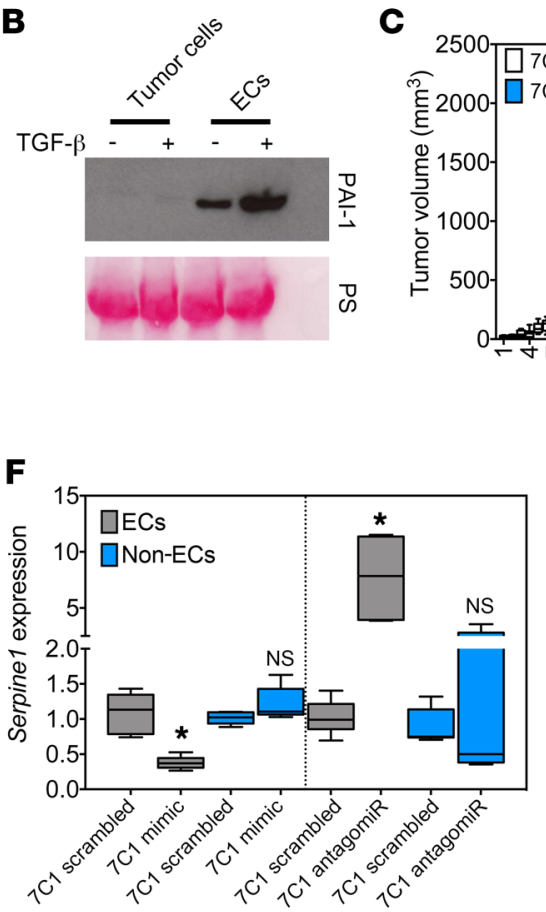

C

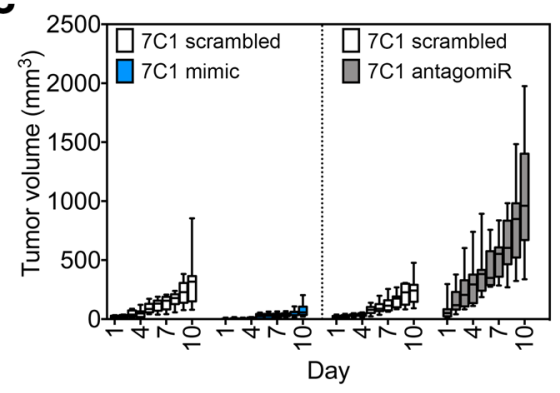

G

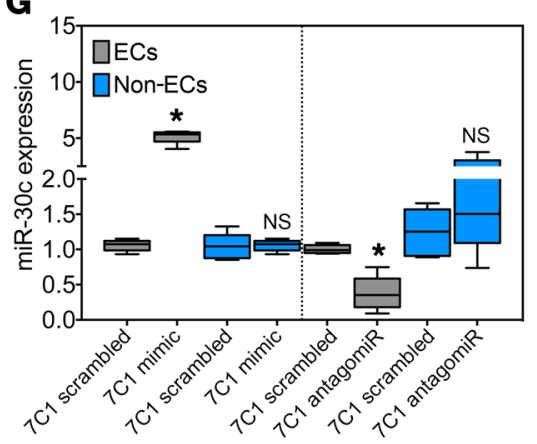

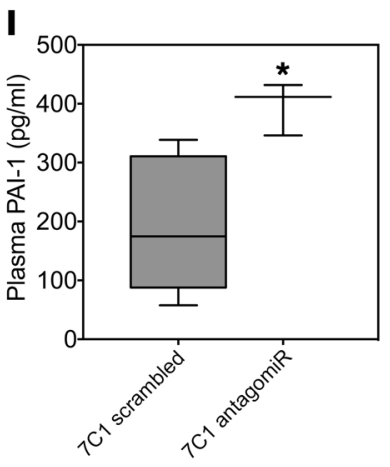

$\mathbf{J}$
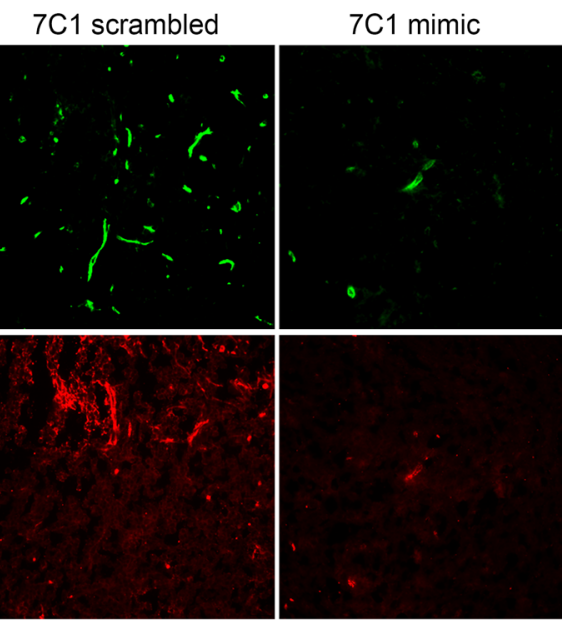

7C1 antagomiR
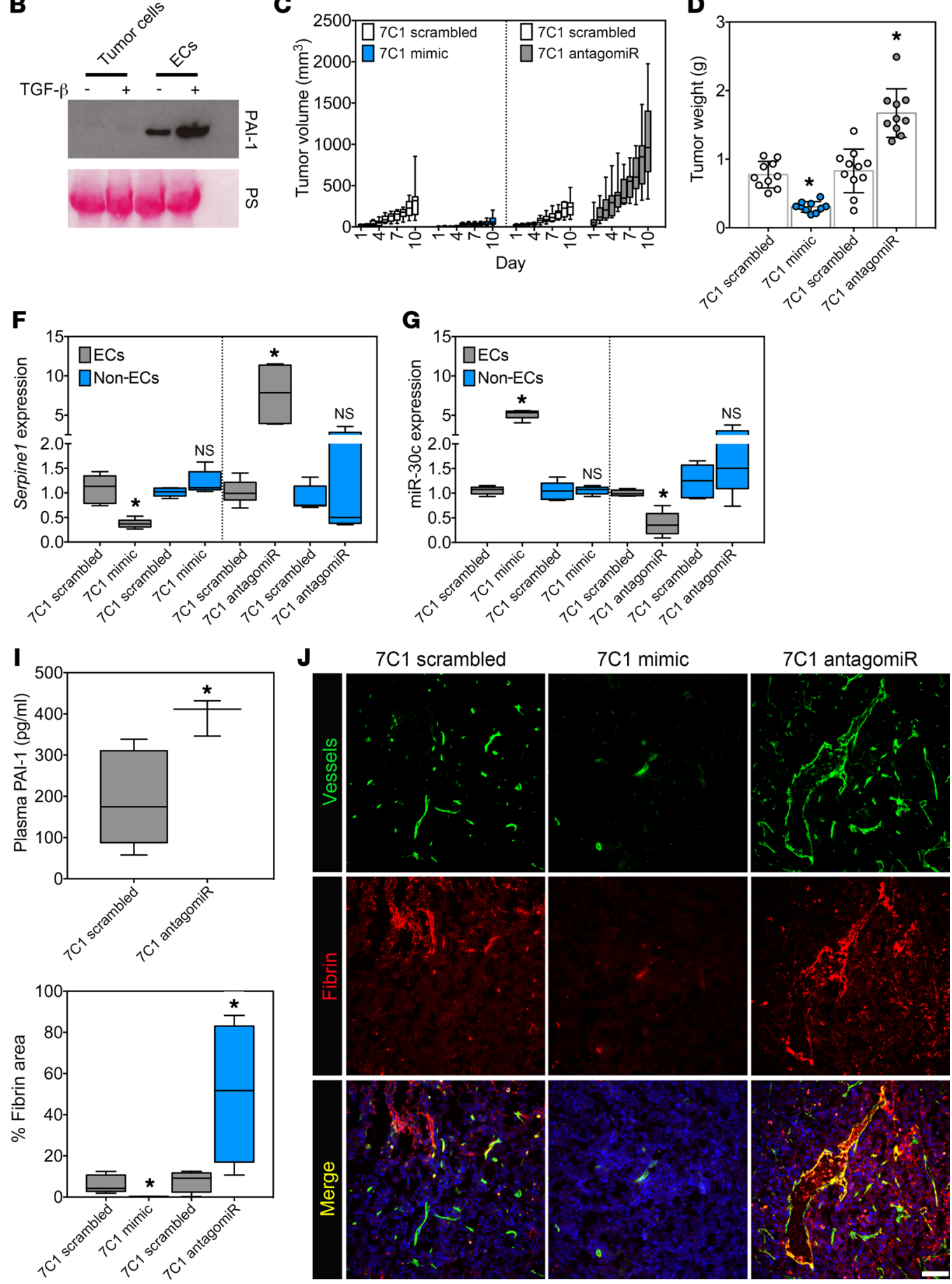

Figure 4. miR-30c gain of function in the endothelium inhibits tumor growth and reduces fibrin abundance, whereas miR-30c loss of function has the opposite effect. (A) qPCR analysis in E0771 mammary tumor cells and ECs treated with $10 \mathrm{ng} / \mathrm{ml}$ TCF- $\beta$ for 48 hours. Samples were assayed in triplicate $(n=3)$. (B) Western blot analysis of PAI-1 in E0771 mammary tumor cells or ECs treated with $10 \mathrm{ng} / \mathrm{ml} \mathrm{TCF-} \beta$ for 48 hours. PS was used to show equal loading. (C) Tumor volume measurements of mice bearing orthotopic E0771 mammary tumors treated intravenously with $1.5 \mathrm{mg} / \mathrm{kg} 7$ 71 scrambled versus $1.5 \mathrm{mg} / \mathrm{kg} 7 \mathrm{C} 1 \mathrm{mimic}$ or $1.5 \mathrm{mg} / \mathrm{kg}$ $7 C 1$ scrambled versus 7C1 antagomiR. Data were combined from 2 independent experiments ( $n=10-11$ mice/group). (D) Tumor weights in mice treated as in C. Data were combined from 2 independent experiments ( $n=10-11$ mice/group). (E) Tumor volume measurements of PAI-1-KO mice bearing orthotopic E0771 mammary tumors treated with 7C1 scrambled, 7C1 mimic, or 7C1 antagomiR ( $n=3-4$ mice/group). (F) qPCR analysis of Serpine1 expression in FACS-sorted ZSGreen ${ }^{+}$ECs and ZSGreen- non-ECs from orthotopic E0771 mammary tumors from mice treated with 7C1 scrambled, 7C1 mimic, or 7C1 antagomiR as in C ( $n=3$ mice/group; $n=2$ replicates). (C) qPCR analysis of miR-30c using FACS-enriched ZSGreen ${ }^{+}$ECs and ZSGreen- non-ECs as in F. (H) Plasma PAI-1 levels in mice treated as indicated ( $n=5$ mice/group). (I) Plasma PAl-1 levels in mice treated as indicated ( $n=5$ mice/group). (J) Immunofluorescence images of E0771 mammary tumors from mice treated with 7C1 scrambled, 7C1 mimic, or 7C1 antagomiR, as above. Nuclei were counterstained with DAPI. Scale bar: $100 \mu \mathrm{m}$. (K) Quantification of vascular area and fibrin deposition area in E0771 mammary tumors from mice treated with 7C1 scrambled, 7C1 mimic, or 7C1 antagomiR ( $n=5$ tumors/group). . $P<0.05$, by Student's $t$ test $(\mathbf{A}, \mathbf{D}, \mathbf{F}-\mathbf{I}$, and $\mathbf{K})$. Data represent the mean \pm SEM. 
by fewer blood vessels and smaller tumors with a pale, avascular appearance when viewed by gross morphology (Supplemental Figure 7A). In contrast, blood vessel densities and fibrin deposition measurements showed a 1.9 -fold increase in vessel area $(4.7 \% \pm 0.3 \%$ versus $8.7 \% \pm 0.8 \%)$ and a 6.5 -fold increase in fibrin deposition $(7.8 \% \pm 2.6 \%$ versus $50.6 \% \pm 17.1 \%)$ in $7 \mathrm{C} 1$ antagomiRtreated mice (Figure 4, J and K). 7C1 antagomiR-treated tumors were superficially bloody in appearance, which is an indicator of increased blood vessel density and/or vascular perturbations (Supplemental Figure 7B). Taken together, both gain and loss of function of miR-30c in ECs using vascular-tropic nanoparticles resulted in a predictable impact on several parameters related to tumor progression: miR-30c overexpression inhibited tumor growth, angiogenesis, and fibrin deposition, while miR-30c antagomiRs produced the opposite result.

Heterogeneous TECs show a spectrum of miR-30c and Serpine1 expression that defines their in vitro sprouting abilities. To further explore the specific role of miR-30c and Serpine1 in the TME, we used FACS to isolate TECs from 2 orthotopic tumor models or from human lung cancer specimens. Using qPCR, we found that miR-30c was expressed by TECs in both murine and human tumors; however, the level of expression varied dramatically between isolates, suggesting that there could be functional heterogeneity in subtypes of TECs based on their intrinsic expression patterns of miR-30c (Figure 5A). To test this possibility and to assess the regulation of miR-30c by TGF- $\beta$ in relation to other putative "angiomiRs," we isolated primary TECs from C3-TAg mammary tumors, a spontaneous tumor model we have previously used to retrieve several TEC isolates for in vitro culture expansion $(39,50)$. A NanoString miRNA array platform was used to measure the abundance of miR30 (and 600 other miRNAs) in TECs challenged with TGF- $\beta$ ex vivo (Figure 5B). Notably, miR-126 was the most abundant miRNA detected, consistent with its designation as an angiomiR that is known to be enriched in the endothelium (51). In accordance with our previous results, TGF- $\beta$ stimulation resulted in the downregulation of miR-30a/-b/-c/-d in TEC cultures (Figure 5B). Additional miRNAs that were increased or decreased by TGF- $\beta$ challenge can be found in the Supplemental NanoString array data file.

To establish links between the expression of miR-30c and functional heterogeneity in the endothelium, we assayed 8 TEC isolates by selecting and expanding $\mathrm{CD} 31^{+} \mathrm{CD} 45^{-}$colonies generated from collagenase-dispersed tumors, as we described previously $(31,39,50)$. Using C3-TAg mouse mammary TECs and KRAS $^{\text {G12D }}$ lung TECs, we again found a spectrum of miR-30c expression across different isolates; i.e., some TECs had high miR30c expression, whereas others had low expression (Figure $5 \mathrm{C}$ ). As predicted, TGF- $\beta$ stimulation resulted in a broad range of Serpine1 induction across these different isolates, with high miR-30c expressers showing the lowest induction of Serpine1 (Figure 5D). The induction of Serpine1 did not appear to relate to the expression of Tgfbr 2 or its coreceptor endoglin, because the expression of these factors was not significantly or consistently associated with the level of Serpine1 expression induced by TGF- $\beta$ (data not shown). Using Pearson's correlation analysis, we found an inverse relationship between the expression of miR-30c and Serpine1 mRNA in TECs from both models, further suggestive of an association between these 2 factors (Supplemental Figure 8A).
To assess the relationship between miR-30c and Serpine1 during EC function in vitro, we simplified the process by designating EC isolates on the basis of their binary expression patterns of miR-30c and Serpine1/PAI-1: miR-30c ${ }^{\text {lo }}$ Serpine $1^{\text {hi }}$ ECs secreted abundant PAI- 1 when challenged with TGF- $\beta$, whereas miR$30 \mathrm{c}^{\text {hi }}$ Serpine $1^{\text {lo }}$ ECs had much lower PAI-1 secretion (Figure 5E). Remarkably, these EC subtypes behaved in an entirely different manner in fibrin bead-sprouting assays. For example, miR-30c ${ }^{\text {lo }}$ PAI- $1^{\text {hi }}$ ECs had an arborescent appearance in fibrin (producing 4.4 \pm 0.19 sprouts/bead over 72 hours), sharply contrasting with their miR-30 $c^{\text {hi }}$ PAI- ${ }^{\text {lo }}$ counterparts, which appeared uncoordinated and dysmorphic in their sprouting abilities and instead produced stunted and immature vascular structures consisting of only $0.1 \pm$ 0.1 sprouts/bead during the same time period (Figure 5, F and G).

TGF- $\beta$ dose-dependently stimulated sprouting in both EC subtypes (though fewer sprouts were present in miR-30 $c^{\text {hi }}$ PAI- $1^{\text {lo }}$ ECs overall) and reduced fibrin degradation as determined with fibrin zymograms (Supplemental Figure 8B and Supplemental Figure 9, A-C). Plating miR-30 $\mathrm{c}^{\mathrm{lo}} \mathrm{PAI}-\mathrm{1}^{\text {hi }}$ ECs on fibrin was sufficient to reduce miR-30c and increase Serpine1 mRNA expression, but this did not occur in ECs from Tgfbr2 ${ }^{2 E C K O}$ mice (Supplemental Figure 9, D and E). Notably, miR-30 ${ }^{\text {hi }}$ PAI- $1^{\text {lo }}$ ECs could be partially induced to form sprouts in different matrices including collagen 1 and Matrigel, indicating that their responses to fibrin-mediated angiogenesis were selectively impaired (Supplemental Figure 10, $A$ and B). These results suggest that the presence of fibrin itself elicits Tgfbr2-dependent activation of the fibrinolytic pathway in ECs that could be driven by autocrine upregulation of TGF- $\beta$ ligands once ECs encounter fibrin matrices. Taken together, we have identified subtypes of ECs in tumors, characterized by a reciprocal expression pattern of $\mathrm{miR}-30 \mathrm{c}$ and Serpine1, that show differential abilities to degrade fibrin and form new vascular structures in vitro (Figure $5 \mathrm{H}$ ).

Identification of Serpine1-enriched TEC subpopulations in vivo using single-cell RNA-Seq. Next, using the 10X Genomics dropletbased sequencing system, we performed single-cell RNA-Seq (scRNA-Seq) of FACS-isolated ZSGreen ${ }^{+}$normal mammary endothelial cells (NECs) or TECs to further assess cellular heterogeneity, particularly with regard to the distribution of genes important for hemostasis and fibrinolysis (Figure 6A). From a total of 2,791 ECs sequenced (1,799 NECs and 992 TECs) and using unsupervised graph-based clustering followed by principal component analysis (PCA) and t-stochastic neighbor embedding (t-SNE), we identified 11 distinct clusters overall (Figure 6B). We performed differential expression analysis of TECs versus NECs, followed by gene set enrichment analysis (GSEA) (52-55). Of note, compared with NECs, TECs showed enrichment for genes with important roles in epithelial-mesenchymal transition (EMT), coagulation, and immune and inflammatory responses (Figure 6C). Selected candidate genes from this analysis are shown in Supplemental Figure 11.

We next reclustered TECs to identify subpopulations on the basis of differential expression of selected genes important for coagulation and fibrinolysis. Within TEC clusters, we found 5 subpopulations (shown as populations 0-4 in the t-SNE plot) (Figure 6D). Interestingly, Serpine1 expression was largely restricted to a single TEC cluster (cluster 1). We also found enrichment for uPar (which binds urokinase, restricts plasminogen activation, 
A

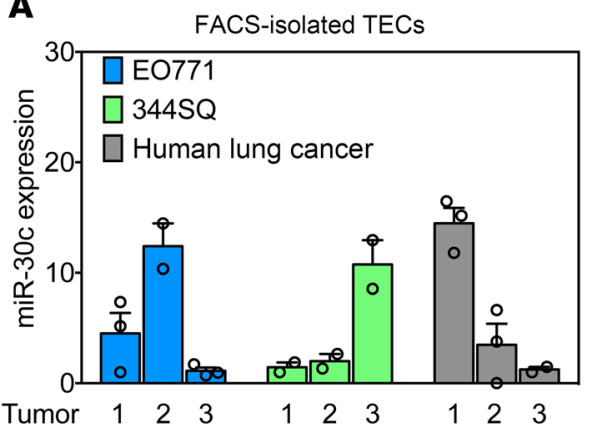

B

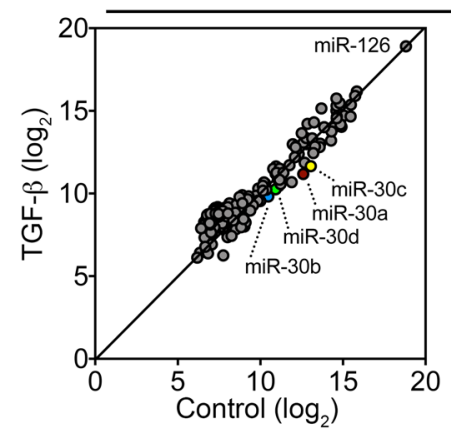

C3-TAg TECs challenged with TGF- $\beta$ in vitro

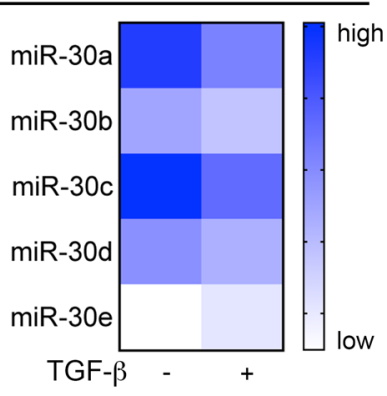

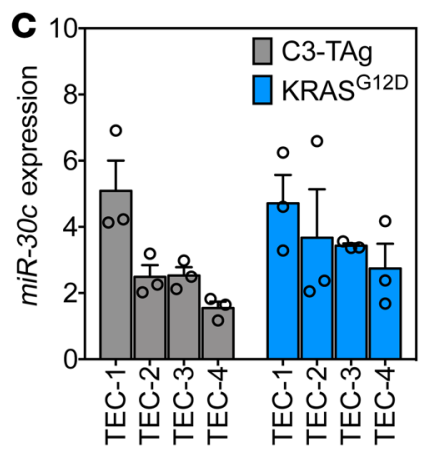

$\mathbf{F}$
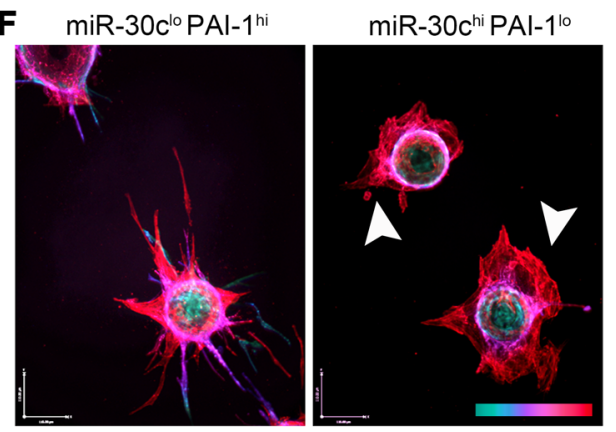

H

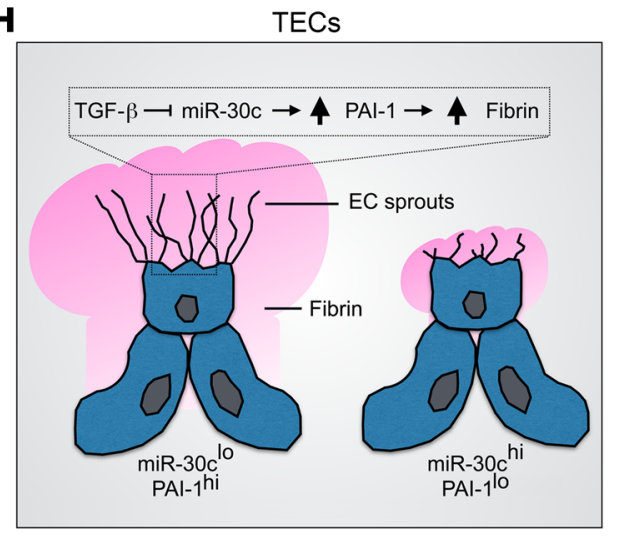

$\mathbf{E}$

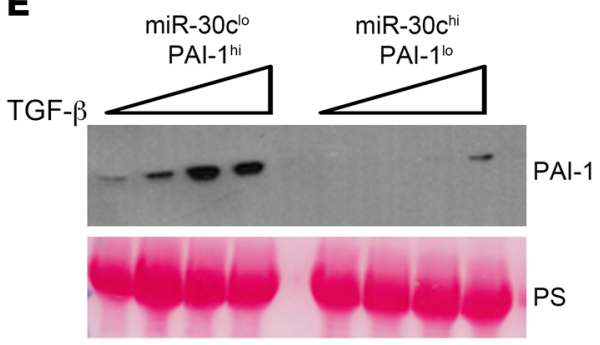

TGF- $\beta-+-+-+-+-+-+-+-+$

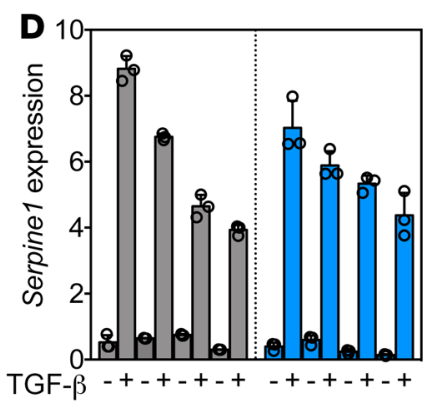

G

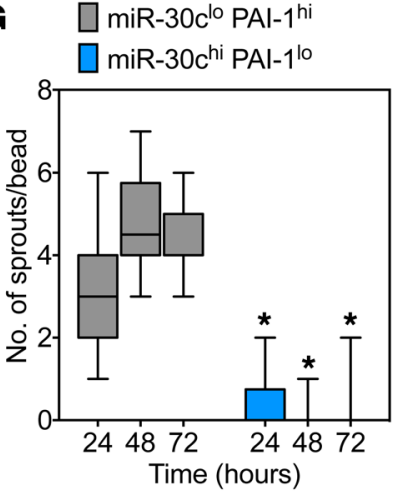

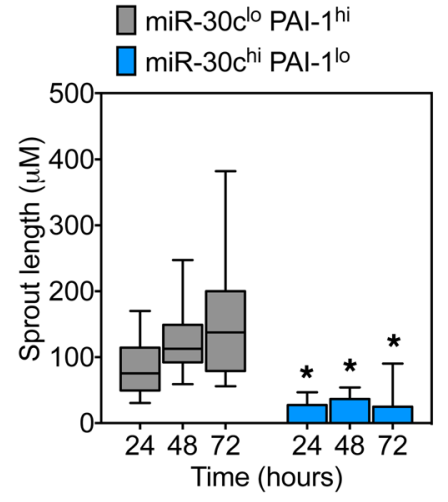

Figure 5. Heterogeneous tumor-associated ECs show a spectrum of miR-30c and Serpine1 expression that defines their in vitro sprouting abilities. (A) qPCR analysis of FACS-enriched TECs from orthotopic E0771 mammary tumors, orthotopic 344SQ lung tumors, and human lung tumor specimens. Samples were assayed in triplicate ( $n=3$ tumors). (B) PCA of NanoString array using TECs treated with $10 \mathrm{ng} / \mathrm{ml} \mathrm{TCF-} \beta$ for 48 hours. A heatmap of miR-30 family members is also shown. (C) qPCR analysis of individual isolates of TECs from the indicated murine tumor model. Samples were assayed in triplicate $(n=3)$. (D) qPCR analysis in C3-TAg TECs or KRAS ${ }^{C 12 D}$ TECs treated with $10 \mathrm{ng} /$ $\mathrm{ml}$ TCF- $\beta$ for 48 hours. Samples were assayed in triplicate. Samples are arranged from low (far left) to high (far right) miR-30c expression on the graph $(n=3)$. (E) Western blot analysis of PAI-1 using conditioned media from miR-30 ${ }^{10}$ PAl- $1^{\text {hi }}$ and miR-30 $c^{\text {hi }}$ PAI- $1^{10}$ ECs treated with TCF- $\beta(0,1,5$, and $10 \mathrm{ng} / \mathrm{ml}$ for 48 hours). PS was used to show equal loading. (F) Images of miR-30c $c^{10} P A l-1^{\text {hi }}$ and $m i R-$ $30 \mathrm{c}^{\text {hi }} \mathrm{PAI}-1^{10} \mathrm{ECs}$ in a fibrin-sprouting assay. Arrowheads indicate aberrant sprout formation in miR$30 \mathrm{c}^{\text {hi }} \mathrm{PAI}-\mathrm{-}^{10} \mathrm{ECs}$. The scale at bottom right indicates sprout depth in the 3D fibrin matrix. Scale bar: $40 \mu \mathrm{m}(x)$ and $54 \mu \mathrm{m}(y)$. (C) Number of sprouts and length of sprouts per bead in miR-30c ${ }^{\text {lo }}$ PAI- $1^{\text {hi }}$ and $\mathrm{miR}-30 \mathrm{c}^{\mathrm{hi}} \mathrm{PAI}-\mathrm{1}^{10} \mathrm{ECs}$. Sprouts were counted at the indicated time points $(n=30$ beads per time point). ${ }^{*} P<0.05$, by ANOVA. (H) Schematic of TEC subtypes showing angiogenic (miR-30 ${ }^{\text {lo }}$ PAI- $1^{\text {hi) }}$ ) versus dysmorphic (miR-30 $c^{\text {hi }} P A I-1^{10}$ ) TECs identified in this study. Data represent the mean \pm SEM. and was previously shown to be expressed in sprouting EC tip cells) and Serbp1 (a relatively unexplored factor suggested to play a role in Serpine1 mRNA stability) within distinct TEC clusters (56). $u P A$ and $t P A$ were expressed at comparatively lower levels overall. Notably, the enrichment of these different genes was not always found within the same TEC clusters, suggesting that subpopulations of TECs are differentially specialized in terms of their ability to coordinate fibrin generation and degradation. For 
A
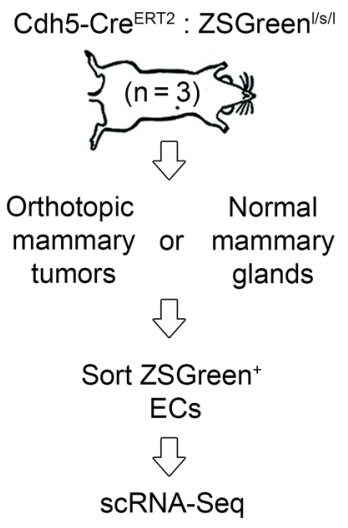

B

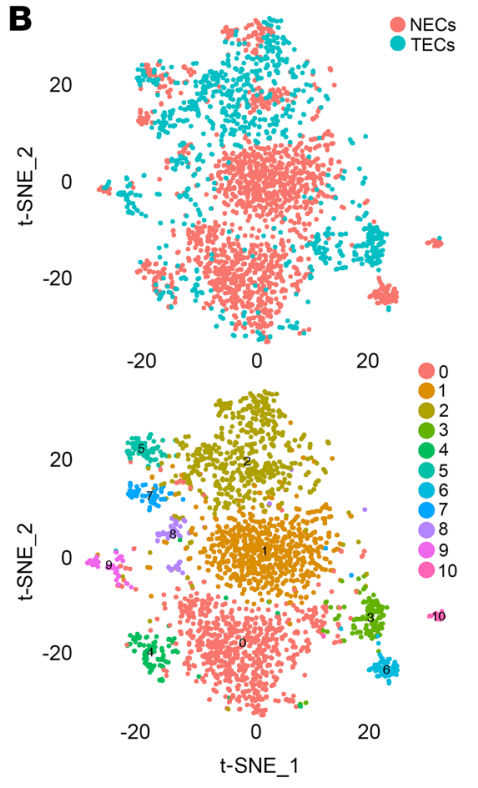

C

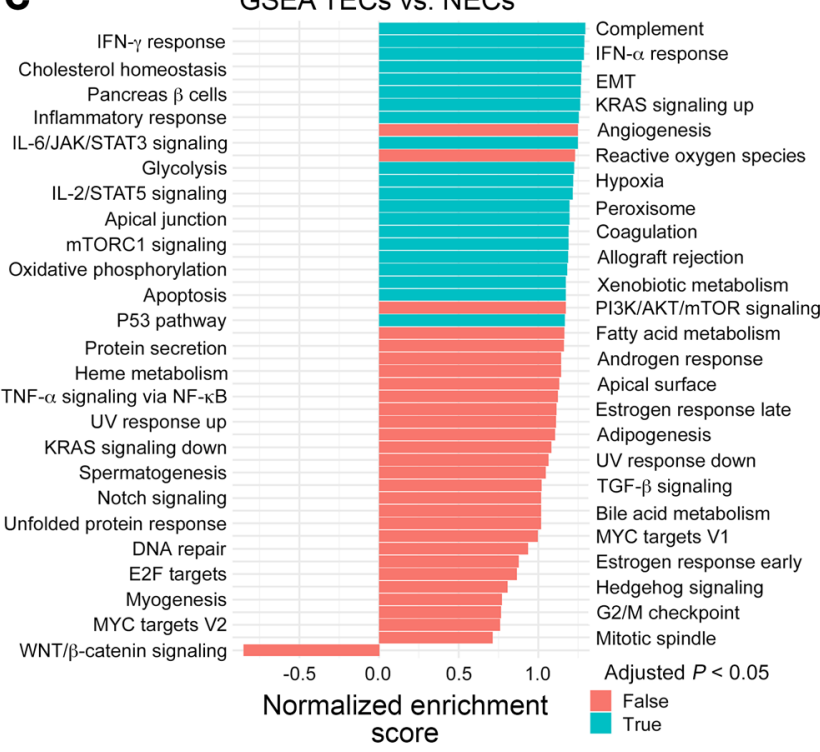

D

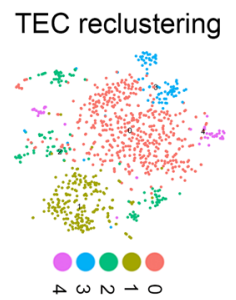

E

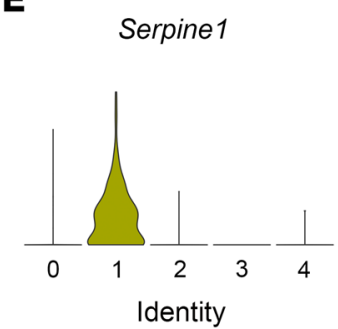

Serpine1
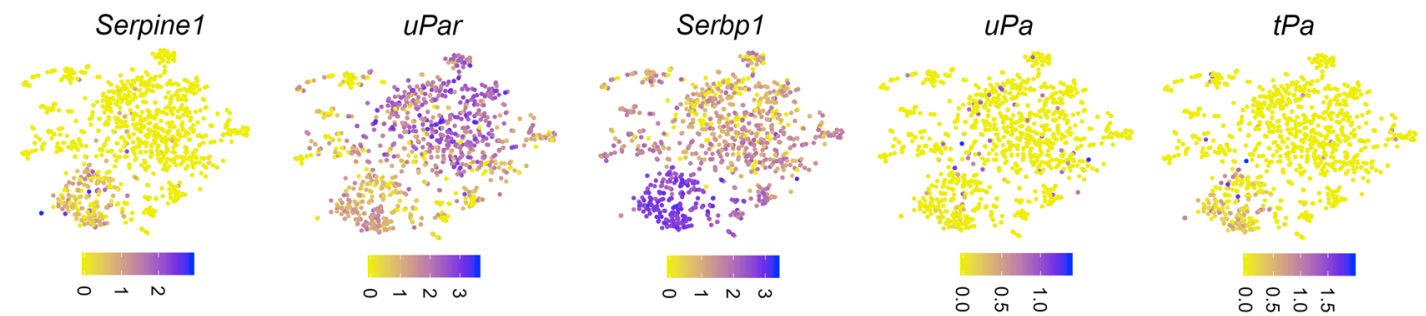

$\mathbf{F}$

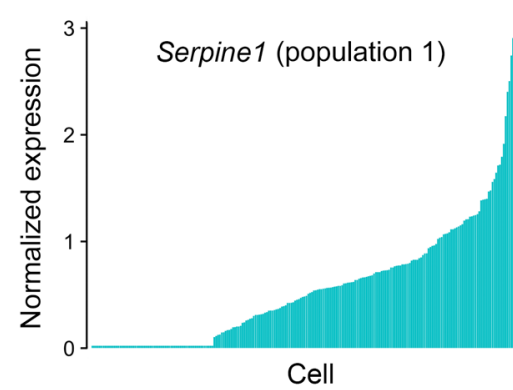

Figure 6. Identification of Serpine1-enriched TEC subpopulations in vivo using scRNA-Seq. (A) Experimental design for isolating ZSCreen ${ }^{+}$NECs or TECs for scRNA-Seq. E0771 mammary tumors or normal mammary glands were harvested (from the contralateral side, $n=3$ individual mice), pooled, and subjected to 10X Genomics scRNA-Seq. (B) t-SNE plots showing clustering of NECs versus TECs. (C) GSEA showing hallmark pathways in TECs versus NECs as determined from scRNA-Seq data. (D) TEC reclustering and t-SNE plots showing enrichment and expression of 5 coagulation and fibrinolysis genes in TEC subpopulations. Serpine1 expression was mostly restricted to population 1. (E) Violin plots showing differential enrichment of Serpine1, uPar, and Serbp1 across 5 TEC subpopulations. (F) Serpine1 expression by individual cell in TEC subpopulation 1 showing a spectrum of Serpine1.

example, plotting their expression across the 5 subpopulations or plotting Serpine1 by individual cells from selected subpopulations showed a broad spectrum of expression (Figure 6, E and F). Thus, there are several genes of known importance during the regulation of coagulation and fibrinolysis enriched in TECs, but their expression is compartmentalized and highly variable within distinct cell subpopulations.

The balance between miR-30c and Serpine1 controls vasculardirected fibrinolysis and angiogenesis. We next took advantage of the EC subtypes uncovered in this study to further explore how a miR-30c/Serpine1 axis controls the rate of vascular-directed fibrinolysis and tumor-associated blood vessel formation. After con- firming that miR-30c antagomiRs or miR-30c mimics would either promote or block PAI- 1 secretion, respectively, we tested the direct role of miR-30c during sprouting angiogenesis in these EC subtypes (Figure 7A). Strikingly, we found that blocking miR-30c in miR-30 chi PAI-1 ${ }^{\text {lo }}$ ECs was sufficient to partially rescue their aberrant sprouting abilities in fibrin, whereas miR-30c mimics reduced in vitro sprouting in the miR-30 $\mathrm{c}^{\mathrm{lo}}$ Serpine $1^{\mathrm{hi}}$ subtype (Figure 7, B and C). Notably, these EC subtypes were advantageous for further confirming the activity of 7C1 nanoparticles coupled to miR-30c mimics or miR-30c antagomiRs, both of which produced sustained effects on PAI-1 secretion and in vitro fibrinolysis as determined using fibrin zymograms (Supplemental Figure 12, A-D). 
A
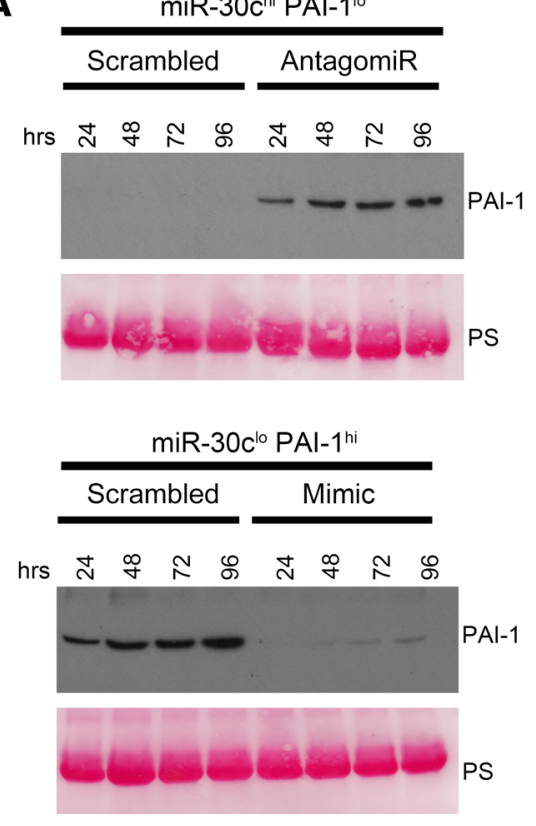

B

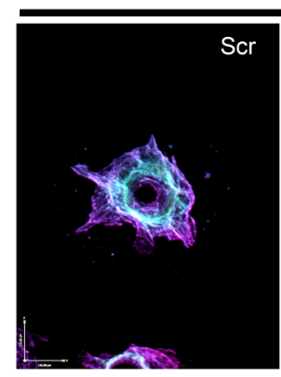

C

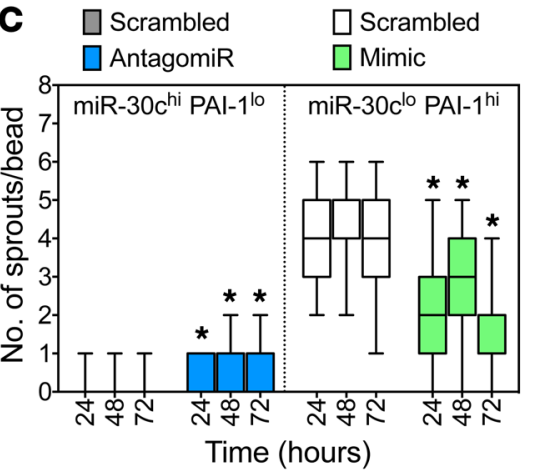

miR-30c $\mathrm{c}^{\mathrm{O}} \mathrm{PAl}-1^{\mathrm{hi}}$
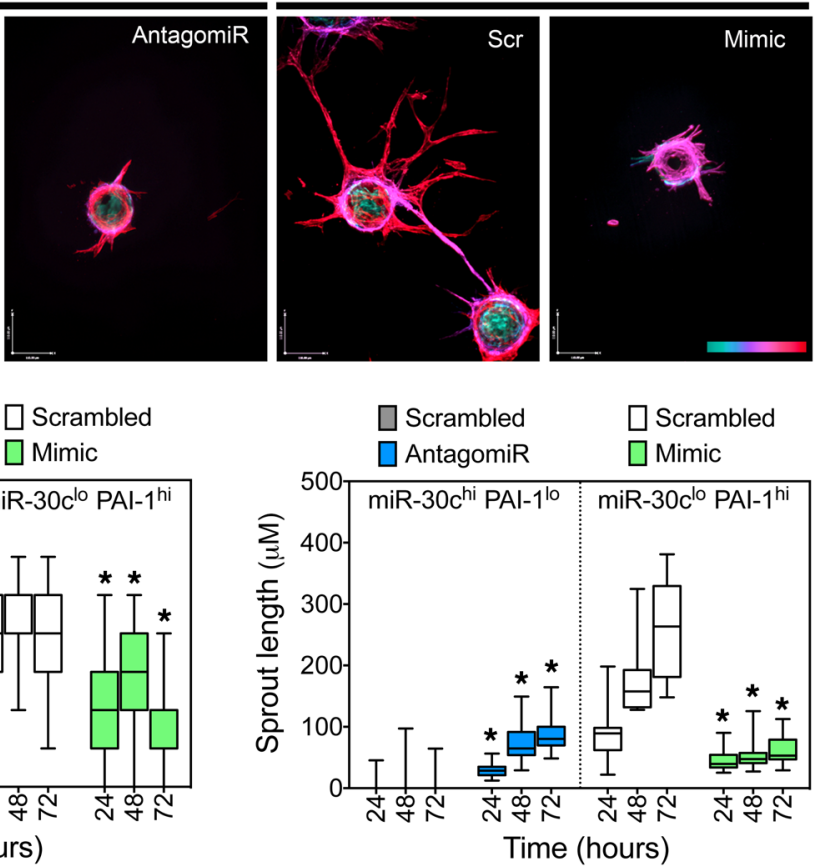
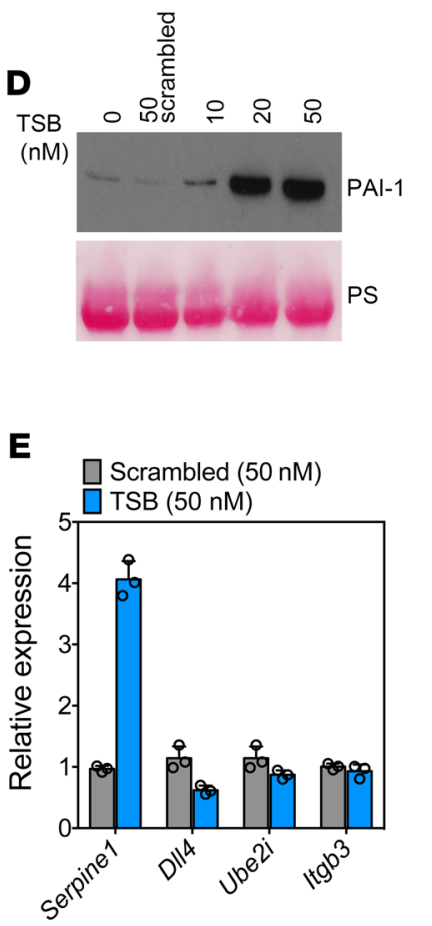

$\mathbf{F}$

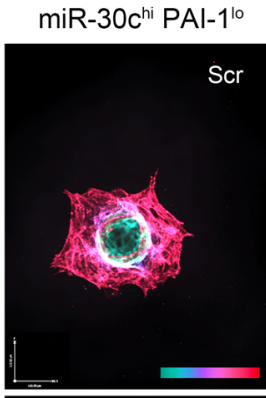

TSB
G

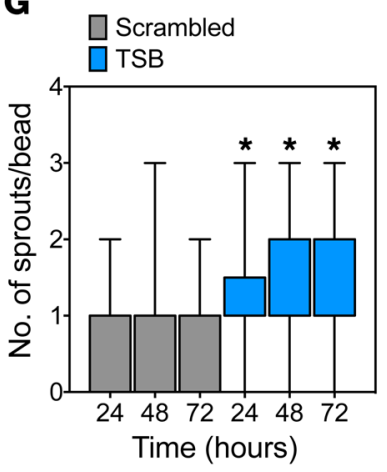

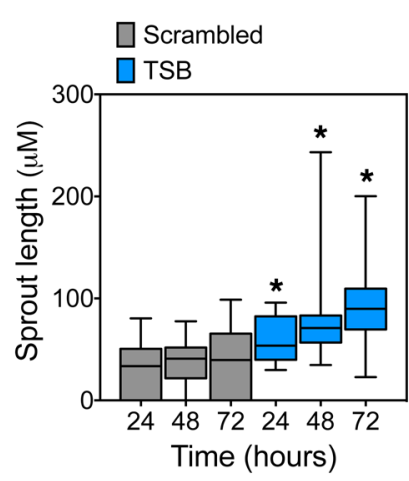

Time (hours)

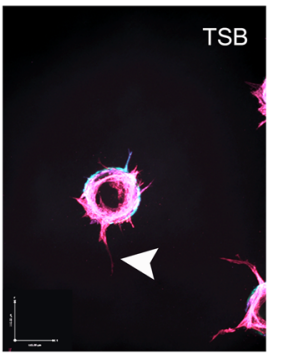

Figure 7. The balance between miR-30c and Serpine1 controls vascular-directed fibrinolysis and angiogenesis. (A) Western blot for PAI-1 using conditioned media from miR-30c $\mathrm{ch}^{\text {hi }} \mathrm{PAI}-\mathrm{-}^{10} \mathrm{ECs}$ treated with $20 \mathrm{nM}$ miR-30c antagomiR or miR-30c $\mathrm{c}^{10}$ PAl- $1^{\text {hi }}$ ECs treated with $20 \mathrm{nM}$ miR-30c mimic. Cells were treated for the indicated durations before harvesting the culture medium. (B) Representative images of vascular structures (fibrin bead assay) in miR-30c $\mathrm{c}^{\mathrm{hi}} \mathrm{PAI}-1^{10} \mathrm{ECs}$ treated with $20 \mathrm{nM}$ miR-30c antagomiR and in miR-30c ${ }^{10} \mathrm{PAl}-1^{\text {hi }} \mathrm{ECs}$ treated with $20 \mathrm{nM}$ miR-30c mimic. Images were captured after 48 hours. The scale at bottom right indicates sprout depth in the 3D fibrin matrix. Scale bars: $40 \mu \mathrm{m}(x)$ and $54 \mu \mathrm{m}(y)$. (C) Number of sprouts per bead and average sprout length of miR-30c hi PAI-1/0 ECs treated with $20 \mathrm{nM}$ miR-30c antagomiR and miR-30 $\mathrm{c}^{10} \mathrm{PAI}-1^{\mathrm{hi}} \mathrm{ECs}$ treated with $20 \mathrm{nM}$ miR-30c mimic. Sprouts were counted and measured at the indicated time points ( $n=30$ beads per condition). Results were analyzed using ANOVA. (D) Western blot using conditioned media from miR-30 $\mathrm{c}^{\text {hi }}$ PAI- $-^{10}$ ECs treated with the indicated doses of TSB for 48 hours. PS was used to show equal loading. (E) qPCR for other miR-30c targets in TSB-treated ECs. Samples were assayed in triplicate. (F) Representative images of vascular structures (fibrin bead assay) in miR-30 $\mathrm{hhi}^{\mathrm{hi}} \mathrm{PAI}-\mathrm{-}^{10} \mathrm{ECs}$ treated with $20 \mathrm{nM}$ TSB or $20 \mathrm{nM}$ scrambled TSB control. Images were captured after 48 hours. Arrowhead indicates an EC sprout. Color scale indicates sprout depth in the 3D fibrin matrix. Scale bars: $40 \mu \mathrm{m}(x)$ and $54 \mu \mathrm{m}(y)$. (C) Number of sprouts per bead and average sprout length in miR-30c ${ }^{\text {hi }}$ PAI- $1^{10} \mathrm{ECs}$ treated with $20 \mathrm{nM}$ TSB or $20 \mathrm{nM}$ scrambled TSB control ( $n=30$ beads per condition). ${ }^{*} P<0.05$, by ANOVA (C and $\mathbf{G}$ ). Data represent the mean \pm SEM. 
A

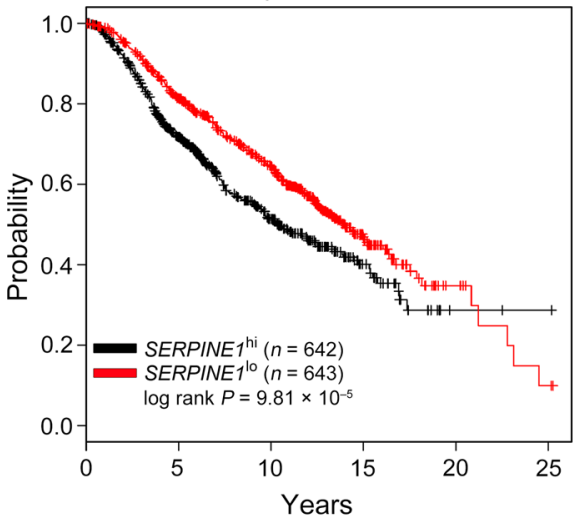

D SERPINE1 expression in breast cancer

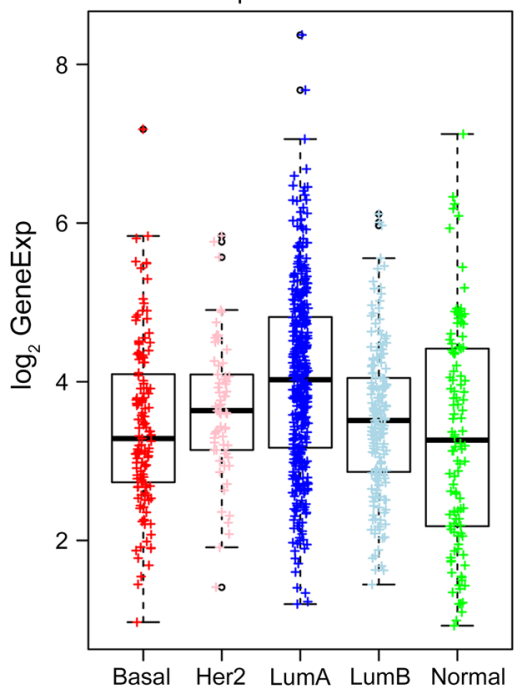

B

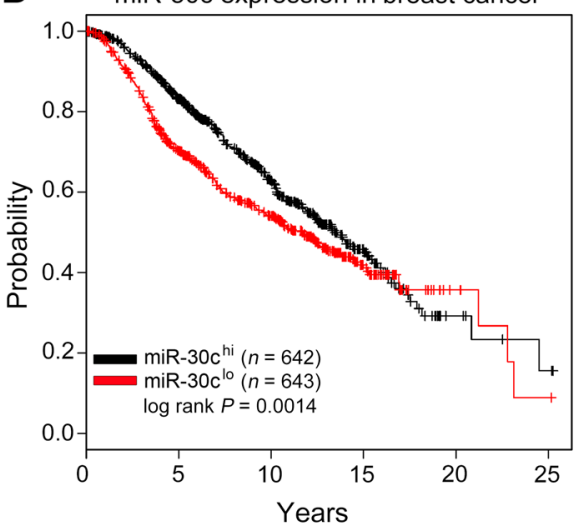

miR-30c expression in breast cancer

C SERPINE1 + miR-30c in breast cancer

E

E $\quad$ miR-30c expression in breast cancer

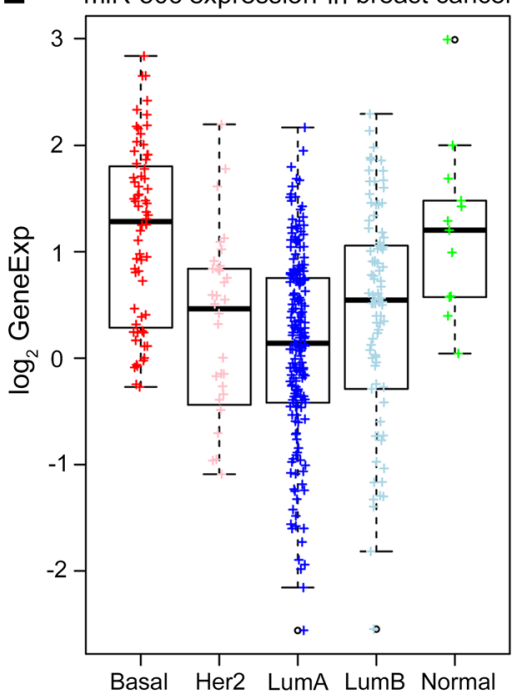

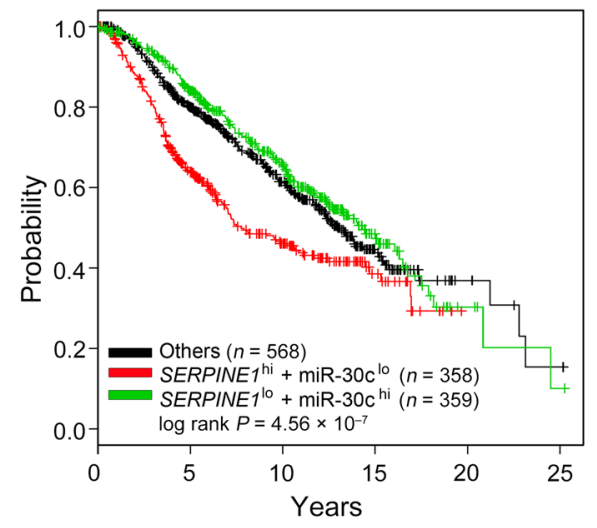

Figure 8. Concurrent miR-30c $\mathrm{c}^{\mathrm{o}}$ Serpine $\mathrm{T}^{\mathrm{hi}}$ expression cumulatively predicts decreased patient survival in a large cohort of breast cancer patients. (A) METABRIC data were downloaded from the Sage BioNetworks Synapse database (https://www.synapse.org/\#!Synapse:syn1688369/wiki/27311). mRNA and miRNA expression data and clinical overall survival data from approximately 1285 patients were examined. SERPINE1 expression in a large cohort of breast cancer patients from a METRABRIC data set is shown. SERPINE1 ${ }^{\text {hi }}$ is defined as patients with SERPINE1 expression higher than the median SERPINE1 expression level. SERPINE1 ${ }^{10}$ is defined as patients with SERPINE1 expression levels lower than or equal to the median SERPINE1 expression level. (B) miR-30c expression in breast cancer patients from the METABRIC data set. Definitions similar to those described above for SERPINE1 were used for patients' miR-30 $\mathrm{c}^{\mathrm{hi}}$ and miR-30 $\mathrm{c}^{10}$ expression levels. (C) Concurrent expression of miR-30c and SERPINE1 in patients with breast cancer from the METRABIC data set. SERPINE1 ${ }^{10} \mathrm{miR}-30 \mathrm{c}^{\mathrm{hi}}$ is defined as patients with SERPINE1 expression lower than the median SERPINE1 expression level and miR-30c expression higher than the median miR-30c expression level. SERPINE ${ }^{\mathrm{hi}} \mathrm{miR}-30 \mathrm{c}^{\mathrm{lo}}$ is defined as patients with SERPINE1 expression higher than the median SERPINE1 expression level and miR-30c expression lower than the median miR-30c expression level. The remaining patients were defined as "others." (D) SERPINE1 expression across breast cancer subtypes in The Cancer Genome Atlas Breast-Invasive Carcinoma (TCGA-BRCA) data set. TCGA breast cancer data were downloaded from the FireBrowse database (http://firebrowse.org/). mRNA and miRNA expression data and PAM50 subtypes were examined. (E) miR-30c expression across breast cancer subtypes in the TCGA-BRCA data set.

miR-30c targets multiple genes that could hypothetically influence vessel sprouting via different mechanisms, thus we used a target site blocker (TSB) to determine the specific role of the miR-30c/Serpine1 axis during fibrin-mediated vessel sprouting. Unlike miR-30c antagomiRs that reduce the interaction of miR-30c with multiple mRNAs, the TSB should only prevent miR-30c from targeting Serpine1, while leaving other miR-30c-mRNA interactions intact. Using the miR-30 $c^{\text {hi }}$ PAI- $1^{\text {1o }}$ EC subtype, we found that a TSB was sufficient to promote PAI-1 secretion and increase Serpine1 mRNA without increasing the expression of other known miR-30c targets including Dll4,
Ube2i, and Itgb3 (Figure 7, D and E). The TSB also partially rescued in vitro sprouting, which was indicated by conversion of an immature and poorly organized cellular "sheet" into a more stereotypical pattern of vessel branches that increased in number and length over time, as is typical of ECs (Figure 7, F and G).

Because we found that fibrin was reduced in tumors from

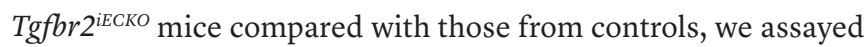
the ability of miR-30c antagomiRs to bypass loss of the TGF- $\beta$ receptor and promote vessel sprouting and fibrin abundance (by inhibiting fibrin degradation) in Tgfbr ${ }^{\text {iECKO }}$ ECs. Consistent with our previous results above, vessel sprouting was diminished 
in ECs from Tgfbr2 ${ }^{i E C K O}$ mice; however, addition of the miR$30 \mathrm{c}$ antagomiR was sufficient to promote vessel sprouting in vitro (Supplemental Figure 13, A and B). Furthermore, miR-30c antagomiRs reduced the robust fibrin degradation in $\mathrm{Tg}_{\mathrm{fbr}} \mathrm{P}^{\text {iECKO }}$ ECs that are ordinarily unresponsive to TGF- $\beta$ (Supplemental Figure 13C). Taken together, our results support the concept that (a) subtypes of ECs in tumors can be functionally stratified on the basis of their expression patterns of miR-30c and Serpine1, and (b) miR-30c plays an important role, downstream of TGF- $\beta$, in balancing fibrin degradation during blood vessel formation.

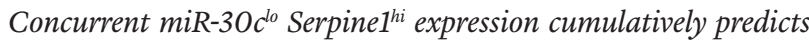
decreased patient survival in a large cohort of breast cancer patients. Finally, because it is challenging to examine the coexpression of miR-30c and/or Serpine1 in human tumor-associated blood vessels in situ and then correlate their expression levels with clinical sequelae, we instead accessed METABRIC (Molecular Taxonomy of Breast Cancer International Consortium) gene expression data (https://www.synapse.org/\#!Synapse:syn1688369/wiki/27311), which includes breast tumor sample and clinical endpoint data from approximately 1200 patients with breast cancer. Of note, breast cancer cells typically do not express PAI-1, or they express very low levels of PAI-1, compared with ECs, as indicated using core biopsies from 12 independent breast tumors found in the publicly available Human Protein Atlas (https://www.proteinatlas.org). Given the identified role for a Serpine1/miR-30c axis in mediating fibrin degradation in the TME of mice, the reported overexpression of SERPINE1 in human breast cancer tissues which correlates with worse overall survival, and the influence of fibrinfibronectin complexes in breast cancer progression, we hypothesized that breast cancer patients with concurrent SERPINE1 ${ }^{\text {hi }}$ miR-30c $c^{\text {lo }}$ expression patterns would have worse overall survival compared with their SERPINE1 ${ }^{\text {lo }}$ miR-30 $c^{\text {hi }}$ counterparts (8). First, we examined survival patterns that might distinguish SERPINE1 ${ }^{\text {hi }}$ versus SERPINE1 ${ }^{\text {lo }}$ and $\mathrm{miR}-30 \mathrm{c}^{\text {hi }}$ versus $\mathrm{miR}-30 \mathrm{c}^{\mathrm{lo}}$ patients with breast cancer. We found that patients with either higher SERPINE1 or lower miR-30c expression had worse overall survival (Figure 8, $\mathrm{A}$ and $\mathrm{B})$. These results are consistent with the recently reported favorable prognostic outcome in a separate cohort of breast cancer patients with miR-30c hi expression relative to their miR-30 ${ }^{\mathrm{lo}}$ counterparts (27). Other miR-30 family members did not show a similar association (data not shown). Notably, patients with higher SERPINE1 expression also had lower miR-30c $\left(r=-0.14, P=8.744^{-07}\right.$, data not shown), and patients with concurrent SERPINE $1^{\text {hi }}$ and miR-30 $c^{\text {lo }}$ coexpression showed a worse survival outcome compared with that of the remaining patients (Figure $8 \mathrm{C}$ ). Thus, there is an improved ability to predict patient outcomes when SERPINE1 and miR-30c expression are examined concurrently as opposed to individually. Finally, we also found that the expression of SERPINE1 and miR-30c mirror one another and is negatively correlated in different cancer subtypes, with SERPINE1 expression being highest in the LumA subtype in parallel with the lowest expression of miR-30c (Figure 8, D and E). Taken together, these results are consistent with a tumor-suppressive function of miR-30c and a tumor-promoting ability of SERPINE1 in the microenvironment of human breast cancer. We propose that these effects are due in part to cooperation between these factors which mediate the rate of perivascular fibrin generation and degradation throughout the TME.

\section{Discussion}

Tumor vasculature, as in wounded vasculature, is typically leaky and hyperpermeable, mainly because of the influence of VEGF, which leads to the escape of plasma proteins such as fibrinogen (57). Although multiple cell types, extracellular matrix (ECM) components, and soluble factors contribute to the woundhealing-like response observed in tumors, seminal observations by Dvorak describe the near-ubiquitous presence of fibrin(ogen) in solid tumors, and later studies demonstrated that fibrin itself is directly proangiogenic $(1,6,58-60)$. Biomechanical forces, driven by thickened sheets of cross-linked ECM in tumors, may also compress the endothelium, leading to vascular injury and plasma leakage (61). The perivascular accumulation of fibrin, along with matrix proteins such as fibronectin, provides scaffolds for new blood vessels (and for cancer cells) and is suggested to be a principal instigator of many of the sequential steps that typify the wound healing-like response observed in solid tumors (7). Thus, how the endothelium senses and responds to the presence of extravascular fibrin is important for vessel homeostasis and vessel maturation as tumors develop. Given its strong transcriptional regulation of the Serpine1 gene, TGF- $\beta$ is central during the coordination of the mainly vascular-driven processes that control the rate of fibrin deposition, fibrin degradation, and tumor progression.

In the present study, we hypothesize that an axis between TGF- $\beta$, miR-30c, and Serpine1 functions as a fibrin-producing pathway in ECs. We demonstrate that a balance in the expression and regulation of these 3 factors controls fibrin abundance and vessel morphogenesis; for example, an imbalance in the expression of miR-30c results in hyperfibrinolytic vessels that are dysmorphic and grow chaotically, and this imbalance is rescued by ectopic expression of a miR-30c antagomiR or a TSB directed to the miR-30c-binding site in the Serpine1 gene. Interestingly, this hyperfibrinolytic phenotype resembles virally transduced ECs that were shown to have enhanced proteolytic activity and form hemangioma-like cystic structures in vitro $(62,63)$. Upregulated protease activity mediated by the UPAR and activation of the TGF- $\beta$ pathway itself in sprouting tip cells have also been described; thus, there appear to be subtypes of ECs positioned throughout the vasculature that have specialized properties that become important during vascular-driven fibrinolysis and sprouting angiogenesis (56). Our results point to an exaggerated wound-healing-like response within these tumor EC subtypes that may relate to selection pressures driven by conditions in the TME (i.e., chronic EC exposure to fibrin and cross-linked ECM) that directly regulate miR-30c expression; alternatively, preexisting EC subtypes may have different abilities to degrade fibrin and form new vascular structures, activities that are related to their intrinsic miR-30c and Serpine1 expression levels.

The pathways that regulate the balance between fibrin deposition and fibrin degradation have long been recognized as important contributors to tumor progression. Patients with breast cancer were shown to have higher levels of circulating D-dimer (a fibrin degradation product) compared with levels in patients with benign breast disease or carcinoma in situ, indicating an active fibrinolytic process in malignant cancers or a systemic activation of coagulation pathways (64). Furthermore, patients with breast or lung cancer with increased PAI-1 in tumors or in serum have 
a worse overall survival and higher incidence of metastasis compared with patients with lower PAI-1 levels $(65,66)$. However, the specific role of PAI-1 during tumor progression, like the presence of intratumoral fibrin(ogen) itself, is controversial and context dependent, especially with regard to tumor angiogenesis (67-69). These discrepancies may relate to the cell type-specific and cancer stage-dependent functions of PAI- 1 within the TME, or may be due to alternative and fibrin-independent roles for PAI-1 in cancer cells, including the ability of PAI-1 to protect against apoptosis and support cell motility via PAI-1-vitronectin interactions (19, 70). Thus, to directly assess how influencing EC-mediated fibrinolysis via miR-30c-regulated PAI-1 would impact tumor growth, we took advantage of vascular tropic nanoparticles, previously shown to efficiently target the vasculature, to deliver miR-30c mimics or miR-30c antagomiRs into WT versus PAI-1-KO mice $(48,49)$. While we demonstrated a clear effect of ectopic miR-30c (or miR-30c antagomiR) on fibrin abundance, vessel morphogenesis, and tumor growth, one limitation was that all blood vessels were targeted rather than tumor blood vessels specifically; however, because we affected a pathway that is probably only activated in sprouting, leaky neovasculature like that found in tumors, our approach is unlikely to impact preexisting vasculature in resting tissues (but will likely affect the wound-healing process). Despite this limitation, we show that manipulating miR-30c in the tumor endothelium phenocopied predicted outcomes related to PAI-1 expression; for example, high circulating PAI-1 levels driven by miR-30c blockade were associated with rapid tumor growth, whereas ectopic miR-30c expression was associated with reduced PAI-1 and slower tumor growth.

A second limitation relates to the use of PAI-1-KO mice in this study. We demonstrate that host PAI-1 was necessary for mediating a response to targeted delivery of $\mathrm{miR}-30 \mathrm{c}$ mimics or antagomiRs but that mammary tumor growth was strongly delayed or inhibited on the PAI-1-KO background, making it challenging to extend these studies over a prolonged period. This growth delay may be related to the almost complete absence of PAI-1 expression in EO771 tumor cells, indicating that our model system is essentially PAI-1 null. It is therefore possible that PAI-1independent effects of $\mathrm{miR}-30 \mathrm{c}$ in the vasculature may appear over time as tumors further develop. Indeed, miR-30c and other miR-30 family members were shown to target additional factors, including the Notch ligand DLL4, that can influence blood vessel sprouting and morphogenesis via different mechanisms $(71,72)$. The targeting of additional pathways by miR-30c in ECs, outside the context of canonical angiogenic signaling, is also possible $(73,74)$. Similarly, plasmin has both fibrin and nonfibrin substrates, and while we demonstrated that inhibiting PAI-1 expression with miR-30c mimics enhanced plasmin activity by fibrin zymograms, we cannot rule out the possibility that plasmin was exerting a tumor growth-inhibitory effect in our model by cleaving additional nonfibrin substrates such as FasL and L1Cam (75). Because of its strong upregulation of PAI-1, our use of the miR-30c antagomiR could similarly protect ECs from FasL-mediated apoptosis, which was previously shown in the context of tumor growth (76). In future studies, it will be interesting to test our strategy for vascular-tropic, nanoparticlemediated delivery of miR-30c mimics using additional tumor cell lines that express endogenous PAI-1 and in different tumor settings in which PAI-1 is operative, including metastatic and premetastatic microenvironments (77).

Finally, we have shown that lower expression of miR-30c in patients with breast cancer predicts a worse prognosis and confirmed that higher expression of SERPINE1 is also a poor prognosis indicator as was previously shown in the European Organization for Research and Treatment of Cancer - Receptor and Biomarker Group (EORTC-RBG) patient cohort ( 8,000 breast cancer patients) (78). We also show that combining these expression signatures resulted in a greater cumulative predictive value relating to overall patient survival. While it is challenging to assign this outcome specifically to the differential functions of TECs in breast tumors that match this particular genetic signature, our results are consistent with a tumor-suppressive role for miR-30c in human breast cancers overall, which we link to its profibrinolytic activity in the TME. Moreover, because SERPINE1 is known to be highly enriched in ECs, it is likely that SERPINE1 mRNA expression in these patients' tumors is largely represented by the tumor vasculature or possibly by other stromal cells such as fibroblasts. Notably, and in solid agreement with our findings, gene expression profiling of microdissected microvasculature from human breast tumors showed that SERPINE1, along with additional gene clusters, can stratify patients on the basis of EC heterogeneity signatures alone (79). Taken together, we have identified what to our knowledge is a previously unrecognized TGF- $\beta$ /PAI-1-dependent and miR-30c-regulated pathway that coordinates vascular-directed fibrinolysis and blood vessel morphogenesis in tumors. Our work thus demonstrates important links between regulation of the fibrinolytic pathway and tumor progression and does so by taking into account the heterogeneous properties of the tumor endothelium.

\section{Methods}

Animals. Tgfbr $2^{f / f l}$ mice were crossed with $\mathrm{Cdh} 5$ - $\mathrm{Cr} \mathrm{e}^{E R T 2}$ to generate

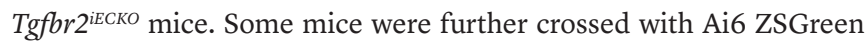
reporter mice to facilitate EC isolation. PAI-1 knockout, Ai6 ZsGreen, $\mathrm{Tgfbr} 2^{f / f l}$, and C57BL/6 control mice were purchased from The Jackson Laboratory at 7 weeks of age. $C d h 5-C r e^{E R T 2}$ mice were generated by Ralf Adams (Max Planck Institute for Molecular Biomedicine). $L S L-K R A S^{G 12 D /+} p 53^{f / f l} L k b 1^{f / / f l}$ mice were provided by Chad Pecot (UNC Chapel Hill). C3-TAg mice were provided by the Mouse Phase 1 Unit at the Lineberger Comprehensive Cancer Center of the University of North Carolina at Chapel Hill.

In vivo animal studies. PAI-1-KO, Cdh5-Cre ${ }^{E R T 2}$, Ai6 ZSGreen, $\mathrm{Tg} f b r 2^{f / f l}$, and control mice were all on a C57BL/6 background. C3-TAg mice were on a friend virus B NIH $(\mathrm{FVB} / \mathrm{N})$ background. $L S L-K R A S^{G 12 D /+} p 53^{f / f l} L k b 1^{f l / f l}$ mice were on a sv129/s4 background. All tumor studies were performed with 8- to 10-week-old mice with a mean weight of 18 to $22 \mathrm{~g}$. Age-matched mice, generated from breeding pairs or ordered directly from The Jackson Laboratory, were randomly allocated to each experimental or control group. Tamoxifen was used to induce deletion of Tgfbr2 and/or induce expression of Cre-controlled genes (e.g., the ZSGreen reporter). Mice at 6 to 7 weeks of age were treated 3 times over the course of 7 days with i.p. injections of $75 \mathrm{mg} / \mathrm{kg}$ tamoxifen. The mice were allowed to recover for 1 week before beginning mammary tumor studies. 
Cell lines. All primary ECs were isolated from normal or tumor tissue from 8- to 12-week-old mice. NECs and TECs from mammary tissue were harvested from female mice. NECs and TECs from lungs were harvested from both male and female mice. Cells were cultured in $1 \mathrm{~g} / 1$ D-glucose DMEM (LG-DMEM) with 10\% FBS, 10\% NuSerum IV, 10 $\mathrm{ng} / \mathrm{ml} \mathrm{VEGF,} 5 \mathrm{ng} / \mathrm{ml} \mathrm{bFGF}$, and $100 \mathrm{mg} / \mathrm{l}$ porcine heparin (referred to here as EC media). E0771 mammary tumor cells were from CH3 BioSystems and were cultured in $4.5 \mathrm{~g} / \mathrm{l} \mathrm{D}$-glucose DMEM (HG-DMEM) and $10 \%$ FBS. Cells were maintained at $37^{\circ} \mathrm{C}$ in $5 \% \mathrm{CO}_{2}$ plus $20 \% \mathrm{O}_{2}$.

Mammary tumor studies in mice. Tgfbr $2^{i E C K O}$ mice, control mice $\left(\mathrm{Tg} f b r 2^{f / f f}\right), \mathrm{C} 57 \mathrm{BL} / 6$ mice, and PAI-1-KO mice were used in the mammary tumor studies. E0771 murine mammary tumor cells were suspended in HBSS at a density of $1.0 \times 10^{7}$ cells $/ \mathrm{ml}$, and $100 \mu \mathrm{l}$ cell suspension was orthotopically injected into the mammary fat pads of 8 - to 10 -week-old mice. Tumors were harvested when they reached $1-2 \mathrm{~cm}^{3}$ in size. Tumor measurements began when tumors were first palpable after injection (usually within 7 to 9 days).

Lung tumor induction in mice. LSL-KRAS $S^{G 12 D /+} p 53^{f / f l} L k b 1^{f / / l}$ mice were anesthetized and then positioned such that their airways were open and unobstructed by their tongue. Once the airway was clear, $37.5 \mu \mathrm{l}$ AdenoCre virus $\left(5 \times 10^{6} \mathrm{PFU} /\right.$ mouse) was added dropwise into the airway of each mouse. The viral inoculation with $37.5 \mu$ l Adeno-Cre was performed twice. Tumors were allowed to grow for 4 weeks before the mice were sacrificed and the tumors harvested. Mice were weighed every 3 to 5 days.

Nanoparticle treatment of mice. Nanoparticle treatment began on the same day as tumor injections in mammary fat pads. Mice were treated 3 times per week intravenously at $1.5 \mathrm{mg} / \mathrm{kg}$. All mice were treated 9 times with nanoparticles except for PAI-1-KO mice, which were treated 12 times because of the longer lag period for tumor establishment.

$s c R N A$-Seq. A total of 3 mice were used. EO771 tumors were injected (1 $\times 10^{6}$ total cells) on one side of the mice (orthotopically in the mammary fat pad), and normal mammary glands were harvested from the other side. To obtain a sufficient number of ECs for sequencing, all samples were pooled prior to sorting. FACS-isolated NECs or TECs were counted and subjected to $10 \times$ preparation and medium output sequencing on the NextSeq 500 (Illumina) with an expected yield of 150,000,000 reads. Raw data were processed according to the manufacturer's guidelines using the $10 \times$ Genomics Cell Ranger toolkit to create FASTQ files. Reads were aligned with STAR against the mm10 genome. Filtered gene-barcode matrices were imported into Rusing the Seurat package, keeping all genes expressed in at least 5 cells and all cells with at least 200 detectable expressed genes. We used a global scaling normalization that normalizes gene expression measurements for each cell by the total expression and log-transforms the result, followed by identification of highly variable genes to use for downstream analyses. Furthermore, we used the canonical correlation analysis-based (CCA-based) approach to "align" the data sets such that gene expression and differential expression could be analyzed without confounding batch effects. The raw data can be accessed on the NCBI's Gene Expression Omnibus (GEO) database (GEO GSE118904).

Statistics. For tumor volumes and weights, the level of significance was determined using ANOVA, Sidek's multiple comparisons test (volumes), or a 2-tailed Student's $t$ test (weights). For branch length, the level of significance was determined using a nonparametric Mann-Whitney $U$ test. All analyses were performed using GraphPad Prism 5 (GraphPad Software). A P value of less than 0.05 was considered significant. All quantitative data represent the mean \pm SEM from at least 3 or more samples or experiments per data point. Additional experimental details (number of animals or cells and experimental replications) are provided in the figure legends. All box and whisker plots show the minimum and maximum values and a horizontal line at the mean.

Study approval. All clinical samples were collected with the patients' consent under a UNC Chapel Hill IRB-approved protocol (IRB no. 14-1755). All animal experiments were carried out in accordance with and under approval of the IACUCs of the University of Virginia and the University of North Carolina at Chapel Hill. Both universities are accredited by the AAALAC International and follow the Public Health Service Policy for the Care and Use of Laboratory Animals. Animal care was provided in accordance with the procedures outlined in the Guide for the Care and Use of Laboratory Animals (National Academies Press, 2011), and protocols were approved by UNC Chapel Hill or the University of Virginia Animal Care and Use Committee.

\section{Author contributions}

JVM and ACD conceived and designed the study. JVM, LX, OFK, PSK, DGA, CVP, ASW, NM, and ACD developed the study methodology. JVM, LX, DJK, CVP, SHA, JP, YST, ASW, KT, NM, and ACD acquired, analyzed, and/or interpreted data. SDT analyzed the scRNA-Seq data set. JVM and ACD wrote the original draft of the manuscript, and all authors wrote, reviewed, and/or revised the manuscript.

\section{Acknowledgments}

ACD is supported by grants from the NIH (RO1-CA177874), the American Cancer Society (129755-RSG-16-176-01-DDC), and an AACR-Bayer Innovation and Discovery Grant (17-80-44-DUDL). Other funding support includes predoctoral fellowships to JVM (F31CA213793-03) and Lin Xiao (AHA 15PRE24470053). This work was partially supported by the United States Army Medical Research and Materiel Command's Armed Forces Institute of Regenerative Medicine (W81XWH-08-2-0034) and MIT's Koch Institute Support (core) Grant P30-CA14051 from the National Cancer Institute (NCI). PSK was supported by a Juvenile Diabetes Research Foundation postdoctoral fellowship (3-PDF-2017-383-A-N). KT is supported by a grant from the Uehara Memorial Foundation. ASW is supported by a grant from the NIH (RO1HL126974). We wish to thank Brian Capaldo (University of Virginia) for assistance with the scRNA-Seq analysis and Scott Hammond (UNC Chapel Hill) for advice regarding analysis of the regulation of miR-30c by TGF- $\beta$.

Address correspondence to: Andrew C. Dudley, Department of Microbiology, Immunology, and Cancer Biology, The University of Virginia, Charlottesville, Virginia 22908, USA. Phone: 434.924.7766; Email: acd2g@virginia.edu.
1. Dvorak HF. Tumors: wounds that do not heal. Similarities between tumor stroma generation and wound healing. N Engl JMed. 1986;315(26):1650-1659.
2. Schäfer M, Werner S. Cancer as an overhealing wound: an old hypothesis revisited. Nat Rev Mol Cell Biol. 2008;9(8):628-638.

3. Farmer P, et al. A stroma-related gene signature predicts resistance to neoadjuvant chemotherapy in breast cancer. Nat Med. 2009;15(1):68-74.

4. Finak G, et al. Stromal gene expression predicts clinical outcome in breast cancer. Nat Med. 
2008;14(5):518-527.

5. Seaman S, Stevens J, Yang MY, Logsdon D, Graff-Cherry C, St Croix B. Genes that distinguish physiological and pathological angiogenesis. Cancer Cell. 2007;11(6):539-554.

6. Brown LF, Van de Water L, Harvey VS, Dvorak HF. Fibrinogen influx and accumulation of crosslinked fibrin in healing wounds and in tumor stroma. Am J Pathol. 1988;130(3):455-465.

7. Nagy JA, Dvorak AM, Dvorak HF. Vascular hyperpermeability, angiogenesis, and stroma generation. Cold Spring Harb Perspect Med. 2012;2(2):a006544.

8. Zhou Z, et al. MRI detection of breast cancer micrometastases with a fibronectin-targeting contrast agent. Nat Commun. 2015;6:7984.

9. Placencio VR, DeClerck YA. Plasminogen activator inhibitor-1 in cancer: rationale and insight for future therapeutic testing. Cancer Res. 2015;75(15):2969-2974.

10. Duffy MJ, McGowan PM, Harbeck N, Thomssen C, Schmitt M. uPA and PAI-1 as biomarkers in breast cancer: validated for clinical use in level-of-evidence-1 studies. Breast Cancer Res. 2014;16(4):428.

11. Masuda T, et al. SK-216, an inhibitor of plasminogen activator inhibitor-1, limits tumor progression and angiogenesis. Mol Cancer Ther. 2013;12:2378-2388.

12. Mashiko S, et al. Inhibition of plasminogen activator inhibitor-1 is a potential therapeutic strategy in ovarian cancer. Cancer Biol Ther. 2015;16(2):253-260

13. Gomes-Giacoia E, Miyake M, Goodison S, Rosser CJ. Targeting plasminogen activator inhibitor-1 inhibits angiogenesis and tumor growth in a human cancer xenograft model. Mol Cancer Ther. 2013;12(12):2697-2708 .

14. Placencio VR, Ichimura A, Miyata T, DeClerck YA. Small Molecule Inhibitors of Plasminogen Activator Inhibitor-1 Elicit Anti-Tumorigenic and Anti-Angiogenic Activity. PLOS ONE. 2015;10(7):e0133786.

15. Takayama $Y$, et al. Inhibition of PAI-1 limits tumor angiogenesis regardless of angiogenic stimuli in malignant pleural mesothelioma. Cancer Res. 2016;76(11):3285-3294.

16. McMahon GA, et al. Plasminogen activator inhibitor-1 regulates tumor growth and angiogenesis. J Biol Chem. 2001;276(36):33964-33968.

17. Almholt K, Nielsen BS, Frandsen TL, Brünner N, Danø K, Johnsen M. Metastasis of transgenic breast cancer in plasminogen activator inhibitor-1 gene-deficient mice. Oncogene. 2003;22(28):4389-4397.

18. Eitzman DT, Krauss JC, Shen T, Cui J, Ginsburg. Lack of plasminogen activator inhibitor-1 effect in a transgenic mouse model of metastatic melanoma. Blood. 1996;87(11):4718-4722.

19. Fang H, Placencio VR, DeClerck YA. Protumorigenic activity of plasminogen activator inhibitor-1 through an antiapoptotic function. J Natl Cancer Inst. 2012;104(19):1470-1484.

20. Bajou K, et al. Absence of host plasminogen activator inhibitor 1 prevents cancer invasion and vascularization. Nat Med.1998;4(8):923-928.

21. Massagué J. How cells read TGF-beta signals. Nat Rev Mol Cell Biol. 2000;1(3):169-178.
22. Dennler S, Itoh S, Vivien D, ten Dijke P, Huet S, Gauthier JM. Direct binding of Smad3 and Smad4 to critical TGF beta-inducible elements in the promoter of human plasminogen activator inhibitor-type 1 gene. EMBO J.1998;17(11):3091-3100.

23. Shi Y, Massagué J. Mechanisms of TGF-beta signaling from cell membrane to the nucleus. Cell. 2003;113(6):685-700.

24. Patel N, Tahara SM, Malik P, Kalra VK. Involvement of miR-30c and miR-301a in immediate induction of plasminogen activator inhibitor- 1 by placental growth factor in human pulmonary endothelial cells. Biochem J. 2011;434(3):473-482.

25. Wu J, et al. Downregulation of microRNA-30 facilitates podocyte injury and is prevented by glucocorticoids. J Am Soc Nephrol. 2014;25(1):92-104.

26. Luo M, et al. Hyperglycaemia-induced reciprocal changes in miR-30 $\mathrm{c}$ and PAI-1 expression in platelets. Sci Rep. 2016;6:36687.

27. Bockhorn J, et al. MicroRNA-30c inhibits human breast tumour chemotherapy resistance by regulating TWF1 and IL-11. Nat Commun. 2013;4:1393.

28. Bockhorn J, et al. MicroRNA-30c targets cytoskeleton genes involved in breast cancer cell invasion. Breast Cancer Res Treat. 2013;137(2):373-382.

29. Chan M, et al. Identification of circulating microRNA signatures for breast cancer detection. Clin Cancer Res. 2013;19(16):4477-4487.

30. Dudley AC, et al. Calcification of multipotent prostate tumor endothelium. Cancer Cell. 2008;14(3):201-211.

31. Xiao L, et al. Tumor endothelial cells with distinct patterns of TGFß-driven endothelialto-mesenchymal transition. Cancer Res. 2015;75(7):1244-1254.

32. Dudley AC. Tumor endothelial cells. Cold Spring Harb Perspect Med. 2012;2(3):a006536.

33. Massagué J. TGF $\beta$ signalling in context. Nat Rev Mol Cell Biol. 2012;13(10):616-630.

34. Bhowmick NA, et al. TGF-beta signaling in fibroblasts modulates the oncogenic potential of adjacent epithelia. Science. 2004;303(5659):848-851.

35. Pepper MS. Transforming growth factor-beta: vasculogenesis, angiogenesis, and vessel wall integrity. Cytokine Growth Factor Rev. 1997;8(1):21-43.

36. Pepper MS, Vassalli JD, Orci L, Montesano R. Biphasic effect of transforming growth factor-beta 1 on in vitro angiogenesis. Exp Cell Res. 1993;204(2):356-363.

37. Ghajar CM, et al. The perivascular niche regulates breast tumour dormancy. Nat Cell Biol. 2013;15(7):807-817.

38. Wang L, et al. Identification of a clonally expanding haematopoietic compartment in bone marrow. EMBO J. 2013;32(2):219-230.

39. Xiao L, McCann JV, Dudley AC. Isolation and culture expansion of tumor-specific endothelial cells. J Vis Exp. 2015;(105):e53072.

40. Levéen $P$, et al. Induced disruption of the transforming growth factor beta type II receptor gene in mice causes a lethal inflammatory disorder that is transplantable. Blood.2002;100(2):560-568.

41. Nakatsu MN, et al. Angiogenic sprouting and capillary lumen formation modeled by human umbilical vein endothelial cells (HUVEC) in fibrin gels: the role of fibroblasts and Angio- poietin-1. Microvasc Res. 2003;66(2):102-112.

42. Blasi F, Carmeliet P. uPAR: a versatile signalling orchestrator. Nat Rev Mol Cell Biol. 2002;3(12):932-943.

43. Carmeliet P. Biomedicine. Clotting factors build blood vessels. Science. 2001;293(5535):1602-1604.

44. Marchand A, Proust C, Morange PE, Lompré AM, Trégouët DA. miR-421 and miR-30c inhibit SERPINE 1 gene expression in human endothelial cells. PLOS ONE. 2012;7(8):e44532.

45. Howe GA, Kazda K, Addison CL. MicroRNA-30b controls endothelial cell capillary morphogenesis through regulation of transforming growth factor beta 2. PLOS ONE. 2017;12(10):e0185619.

46. Thomas MF, L'Etoile ND, Ansel KM. Eri1: a conserved enzyme at the crossroads of multiple RNA-processing pathways. Trends Genet. 2014;30(7):298-307.

47. Ghini F, Rubolino C, Climent M, Simeone I, Marzi MJ, Nicassio F. Endogenous transcripts control miRNA levels and activity in mammalian cells by target-directed miRNA degradation. Nat Commun. 2018;9(1):3119.

48. Dahlman JE, et al. In vivo endothelial siRNA delivery using polymeric nanoparticles with low molecular weight. Nat Nanotechnol. 2014;9(8):648-655

49. Xue W, et al. Small RNA combination therapy for lung cancer. Proc Natl Acad Sci U S A. 2014;111(34):E3553-E3561.

50. Xiao L, Harrell JC, Perou CM, Dudley AC. Identification of a stable molecular signature in mammary tumor endothelial cells that persists in vitro. Angiogenesis. 2014;17(3):511-518.

51. Wang S, Olson EN. AngiomiRs--key regulators of angiogenesis. Curr Opin Genet Dev. 2009;19(3):205-211.

52. Soneson C, Robinson MD. Bias, robustness and scalability in single-cell differential expression analysis. Nat Methods. 2018;15(4):255-261.

53. Subramanian A, et al. Gene set enrichment analysis: a knowledge-based approach for interpreting genome-wide expression profiles. Proc Natl Acad Sci U S A. 2005;102(43):15545-15550.

54. Liberzon A, Birger C, Thorvaldsdóttir H, Ghandi M, Mesirov JP, Tamayo P. The Molecular Signatures Database (MSigDB) hallmark gene set collection. Cell Syst. 2015;1(6):417-425.

55. Sergushichev A. An algorithm for fast preranked gene set enrichment analysis using cumulative statistic calculation. bioRxiv. 2016; https://doi.org/10.1101/060012.

56. del Toro R, et al. Identification and functional analysis of endothelial tip cell-enriched genes. Blood. 2010;116(19):4025-4033.

57. Nagy JA, Dvorak AM, Dvorak HF. VEGF-A and the induction of pathological angiogenesis. Annu Rev Pathol. 2007;2:251-275.

58. Dvorak HF. Rous-Whipple Award lecture. How tumors make bad blood vessels and stroma. Am J Pathol. 2003;162(6):1747-1757.

59. Dvorak HF, Dickersin GR, Dvorak AM, Manseau EJ, Pyne K. Human breast carcinoma: fibrin deposits and desmoplasia. Inflammatory cell type and distribution. Microvasculature and infarction. J Natl Cancer Inst. 1981;67(2):335-345.

60. Brown LF, Dvorak AM, Dvorak HF. Leaky vessels, fibrin deposition, and fibrosis: a sequence 
of events common to solid tumors and to many other types of disease. Am Rev Respir Dis. 1989;140(4):1104-1107.

61. Bordeleau F, et al. Matrix stiffening promotes a tumor vasculature phenotype. Proc Natl Acad Sci U S A. 2017;114(3):492-497.

62. Montesano R, Pepper MS, Möhle-Steinlein U, Risau W, Wagner EF, Orci L. Increased proteolytic activity is responsible for the aberrant morphogenetic behavior of endothelial cells expressing the middle T oncogene. Cell. 1990;62(3):435-445.

63. Dubois-Stringfellow N, Jonczyk A, Bautch VL. Perturbations in the fibrinolytic pathway abolish cyst formation but not capillary-like organization of cultured murine endothelial cells. Blood. 1994;83(11):3206-3217.

64. Blackwell K, et al. Plasma D-dimer levels in operable breast cancer patients correlate with clinical stage and axillary lymph node status. JClin Oncol. 2000;18(3):600-608.

65. Pavey SJ, Hawson GA, Marsh NA. Impact of the fibrinolytic enzyme system on prognosis and survival associated with non-small cell lung carcinoma. Blood Coagul Fibrinolysis. 2001;12(1):51-58. 66. Weigelt B, Peterse JL, van 't Veer LJ. Breast cancer metastasis: markers and models. Nat Rev Cancer. 2005;5(8):591-602.

67. Palumbo JS, Talmage KE, Liu H, La Jeunesse CM, Witte DP, Degen JL. Plasminogen supports tumor growth through a fibrinogen-dependent mechanism linked to vascular patency. Blood. 2003;102(8):2819-2827.

68. Palumbo JS, Potter JM, Kaplan LS, Talmage K, Jackson DG, Degen JL. Spontaneous hematogenous and lymphatic metastasis, but not primary tumor growth or angiogenesis, is diminished in fibrinogen-deficient mice. Cancer Res. 2002;62(23):6966-6972.

69. Bajou K, et al. PAI-1 mediates the antiangiogenic and profibrinolytic effects of $16 \mathrm{~K}$ prolactin. Nat Med. 2014;20(7):741-747.

70. Zhou A, Huntington JA, Pannu NS, Carrell RW, Read RJ. How vitronectin binds PAI-1 to modulate fibrinolysis and cell migration. Nat Struct Biol. 2003;10(7):541-544.

71. Bridge $\mathrm{G}$, et al. The microRNA-30 family targets DLL4 to modulate endothelial cell behavior during angiogenesis. Blood. 2012;120(25):5063-5072.

72. Jiang Q, et al. miR-30a regulates endothelial tip cell formation and arteriolar branching. Hypertension. 2013;62(3):592-598.
73. Duisters RF, et al. miR-133 and miR-30 regulate connective tissue growth factor: implications for a role of microRNAs in myocardial matrix remodeling. Circ Res. 2009;104(2):170-178.

74. Li J, Donath S, Li Y, Qin D, Prabhakar BS, Li P. miR-30 regulates mitochondrial fission through targeting p53 and the dynamin-related protein-1 pathway. PLoS Genet. 2010;6(1):e1000795.

75. Valiente M, et al. Serpins promote cancer cell survival and vascular co-option in brain metastasis. Cell. 2014;156(5):1002-1016.

76. Bajou K, et al. Plasminogen activator inhibitor-1 protects endothelial cells from FasL-mediated apoptosis. Cancer Cell. 2008;14(4):324-334.

77. Psaila B, Lyden D. The metastatic niche: adapting the foreign soil. Nat Rev Cancer. 2009;9(4):285-293.

78. Look MP, et al. Pooled analysis of prognostic impact of urokinase-type plasminogen activator and its inhibitor PAI-1 in 8377 breast cancer patients. J Natl Cancer Inst. 2002;94(2):116-128.

79. Pepin F, et al. Gene-expression profiling of microdissected breast cancer microvasculature identifies distinct tumor vascular subtypes. Breast Cancer Res. 2012;14(4):R120. 